(1)

CrossMark

\title{
Management of severe asthma: a European Respiratory Society/American Thoracic Society guideline
}

Fernando Holguin $\mathbb{1}^{1,34}$, Juan Carlos Cardet (1) ${ }^{2}$, Kian Fan Chung ${ }^{3}$, Sarah Diver ${ }^{4}$, Diogenes S. Ferreira ${ }^{5,6}$, Anne Fitzpatrick ${ }^{7}$, Mina Gaga $\mathbb{0}^{8}$, Liz Kellermeyer $\mathbb{1}^{9}$, Sandhya Khurana ${ }^{10}$, Shandra Knight ${ }^{9}$, Vanessa M. McDonald ${ }^{11}$ Rebecca L. Morgan ${ }^{12}$, Victor E. Ortega ${ }^{13}$, David Rigau ${ }^{14}$, Padmaja Subbarao ${ }^{15}$, Thomy Tonia $^{16}$, Ian M. Adcock ${ }^{17}$, Eugene R. Bleecker ${ }^{18}$, Chris Brightling ${ }^{19}$, Louis-Philippe Boulet ${ }^{20}$, Michael Cabana ${ }^{21}$, Mario Castro ${ }^{22}$, Pascal Chanez ${ }^{23}$, Adnan Custovic ${ }^{24}$, Ratko Djukanovic ${ }^{25}$, Urs Frey ${ }^{26}$, Betty Frankemölle ${ }^{27}$, Peter Gibson (1028, Dominique Hamerlijnck (1027, Nizar Jarjour ${ }^{29}$, Satoshi Konno ${ }^{30}$, Huahao Shen ${ }^{31}$, Cathy Vitary ${ }^{32}$ and Andy Bush ${ }^{33,35}$

@ERSpublications

The ERS/ATS Task Force makes recommendations on the use of novel therapies for severe asthma, specifically biologicals for type 2 high asthma, and antimuscarinic agents and macrolides, as well as on biomarkers for predicting treatment response http://bit.ly/2kZLRaD

Cite this article as: Holguin F, Cardet JC, Chung KF, et al. Management of severe asthma: a European Respiratory Society/American Thoracic Society guideline. Eur Respir J 2020; 55: 1900588 [https://doi.org/ 10.1183/13993003.00588-2019].

ABSTRACT This document provides clinical recommendations for the management of severe asthma. Comprehensive evidence syntheses, including meta-analyses, were performed to summarise all available evidence relevant to the European Respiratory Society/American Thoracic Society Task Force's questions. The evidence was appraised using the GRADE (Grading of Recommendations, Assessment, Development and Evaluation) approach and the results were summarised in evidence profiles. The evidence syntheses were discussed and recommendations formulated by a multidisciplinary Task Force of asthma experts, who made specific recommendations on six specific questions. After considering the balance of desirable and undesirable consequences, quality of evidence, feasibility, and acceptability of various interventions, the Task Force made the following recommendations: 1) suggest using anti-interleukin (IL)-5 and anti-IL5 receptor $\alpha$ for severe uncontrolled adult eosinophilic asthma phenotypes; 2) suggest using a blood eosinophil cut-point $\geqslant 150 \mu \mathrm{L}^{-1}$ to guide anti-IL-5 initiation in adult patients with severe asthma; 3) suggest considering specific eosinophil $\left(\geqslant 260 \mu \mathrm{L}^{-1}\right)$ and exhaled nitric oxide fraction $(\geqslant 19.5 \mathrm{ppb})$ cut-offs to identify adolescents or adults with the greatest likelihood of response to anti-IgE therapy; 4) suggest using inhaled tiotropium for adolescents and adults with severe uncontrolled asthma despite Global Initiative for Asthma (GINA) step 4-5 or National Asthma Education and Prevention Program (NAEPP) step 5 therapies; 5) suggest a trial of chronic macrolide therapy to reduce asthma exacerbations in persistently symptomatic or uncontrolled patients on GINA step 5 or NAEPP step 5 therapies, irrespective of asthma phenotype; and 6) suggest using anti-IL-4/13 for adult patients with severe eosinophilic asthma and for those with severe corticosteroid-dependent asthma regardless of blood eosinophil levels. These recommendations should be reconsidered as new evidence becomes available.

This article has supplementary material available from erj.ersjournals.com

Received: 13 April 2019 | Accepted after revision: 08 Aug 2019

Copyright CERS 2020 


\section{Introduction}

The first European Respiratory Society (ERS)/American Thoracic Society (ATS) guidelines on severe asthma in adults and school age children were published in 2014 [1]. Severe asthma was defined as [1]:

When a diagnosis of asthma is confirmed and comorbidities addressed, severe asthma is defined as "asthma that requires treatment with high dose inhaled corticosteroids [...] plus a second controller (and/or systemic corticosteroids) to prevent it from becoming 'uncontrolled' or which remains 'uncontrolled' despite this therapy'.

Emphasis was placed on the necessity to confirm the diagnosis of asthma and exclude other conditions that may mimic asthma. In addition, the guidelines recognised that severe asthma is a heterogeneous condition consisting of phenotypes such as severe eosinophilic asthma, and specific recommendations were made on the use of sputum eosinophil count and exhaled nitric oxide fraction $\left(F_{\mathrm{ENO}}\right)$ to guide therapy. Recommendations were also made for the use of methotrexate, macrolide antibiotics, antifungal agents, bronchial thermoplasty and the anti-IgE antibody omalizumab in severe asthma.

This current guideline, for which work commenced in 2017, is also an ERS/ATS collaboration and was initiated in view of the rapid introduction of new treatments for severe asthma, particularly the new biological treatments approved for the management of severe eosinophilic asthma. Six specific and important questions were formulated using the PICO (Patient population, Intervention, Comparison and Outcome) format. The GRADE (Grading of Recommendations, Assessment, Development and Evaluation) approach was used to assess the strength of evidence and develop recommendations [2].

The six questions chosen and developed by the Task Force are shown in table 1.

During the deliberations of the Task Force, it became clear that the interleukin- 4 receptor $\alpha$ (IL-4R $\alpha$ ) blocker dupilumab, which modulates the effects of IL-4 and IL-13, would receive approval by the regulatory authorities, so the sixth PICO was instituted, having originally not been considered. The current Task Force was focused on these specific PICOs and, unlike the first Task Force, did not consider general management strategies for severe asthma.

\section{Methods}

A detailed description of the methodology used to develop the questions, rate the outcomes, select the studies, and synthesise, formulate and grade the evidence is available in previous ERS/ATS guidelines and in the supplementary material $[3,4]$.

Affiliations: ${ }^{1}$ Pulmonary Sciences and Critical Care Medicine, University of Colorado, Denver, CO, USA ${ }^{2}$ Allergy and Immunology, University of South Florida, Tampa, FL, USA. ${ }^{3}$ Experimental Studies Medicine, National Heart and Lung Institute, Imperial College London, London, UK. ${ }^{4}$ Respiratory Biomedical Unit, University of Leicester, Leicester, UK. ${ }^{5}$ Alergia e Imunologia, Complexo Hospital de Clinicas, Universidade Federal do Parana, Curitiba, Brazil. 'School of Public Health and Preventive Medicine, Monash University, Melbourne, Australia. ${ }^{7}$ Division of Pulmonology Allergy/Immunology, Cystic Fibrosis and Sleep, Emory University, Atlanta, GA, USA. ${ }^{8}$ Respiratory Medicine Dept and Asthma Centre, Athens Chest Hospital, Athens, Greece. ${ }^{9}$ Biomedical Library, National Jewish Health, Denver, CO, USA. ${ }^{10}$ Pulmonary Diseases and Critical Care, University of Rochester, Rochester, NY, USA. ${ }^{11}$ School of Nursing, University of Newcastle, Newcastle, Australia. ${ }^{12}$ Health Research Methods, Evidence and Impact, McMaster University, Hamilton, ON, Canada. ${ }^{13}$ Pulmonary, Critical Care, Allergy and Immunologic Diseases, Wake Forest School of Medicine, WinstonSalem, NC, USA. ${ }^{14}$ Iberoamerican Cochrane Centre, Barcelona, Spain. ${ }^{15}$ Dept of Pediatrics, SickKids, Toronto, ON, Canada. ${ }^{16}$ Institute of Social and Preventive Medicine, University of Bern, Bern, Switzerland. ${ }^{17}$ Molecular Cell Biology Group, National Heart and Lung Institute, Imperial College of London, London, UK. ${ }^{18}$ Division of Genetics, Genomics and Precision Medicine, University of Arizona, Tucson, AZ, USA. ${ }^{19}$ Dept of Respiratory Sciences, University of Leicester, Leicester, UK. ${ }^{20}$ Respiratory Medicine, Laval University, Quebec, QC, Canada. ${ }^{21}$ Division of General Pediatrics, University of California San Francisco, San Francisco, CA, USA. ${ }^{22}$ Division of Pulmonary and Critical Care Medicine, Washington University, St Louis, MO, USA. ${ }^{23}$ Dept of Respiratory Diseases, University of Aix-Marseille, Marseille, France. ${ }^{24}$ Paediatric Allergy, National Heart and Lung Institute, Imperial College of London, London, UK. ${ }^{25}$ Respiratory Biomedical Research, University of Southampton, Southampton, UK. ${ }^{26}$ Dept of Pediatrics, University Children's Hospital, Basel, Switzerland. ${ }^{27}$ European Lung Foundation, Lausanne, Switzerland. ${ }^{28} \mathrm{School}$ of Medicine and Public Health, University of Newcastle, Newcastle, Australia. ${ }^{29}$ Division of Pulmonary and Critical Care, University of Wisconsin, Madison, WI, USA. ${ }^{30}$ Dept of Respiratory Medicine, Faculty of Medicine and Graduate School of Medicine, Hokkaido University, Hokkaido, Japan. ${ }^{31}$ Dept of Respiratory and Critical Care Medicine, The Second Affiliated Hospital of Zhejiang University School of Medicine, Hangzhou, China. ${ }^{32}$ Asthma Institute, University of Pittsburgh, Pittsburgh, PA, USA. ${ }^{33}$ Dept of Paediatrics, Imperial College London, National Heart and Lung Institute, London, UK. ${ }^{34} \mathrm{~F}$. Holguin is ATS co-chair. ${ }^{35} \mathrm{~A}$. Bush is ERS co-chair.

Correspondence: Fernando Holguin, CU Anschutz Research Complex II, 12700 East 19th Avenue, 9C03, Aurora, CO 80045, USA. E-mail: fernando.holguinducdenver.edu 
TABLE 1 European Respiratory Society (ERS)/American Thoracic Society (ATS) Severe Asthma Task Force questions

1 Should a monoclonal anti-IL-5 antibody be used in adults and children\# with severe asthma?

2 Should a measurement of a specific biomarker be used to guide initiation of treatment with a monoclonal anti-IL-5 or IL-5R $\alpha$ antibody in adults and children with severe asthma? (Chosen biomarkers being $F_{\text {ENO }}$, peripheral or sputum eosinophils and serum periostin)

3 Should a measurement of a specific biomarker be used, in addition to total IgE level, to guide initiation of treatment with a monoclonal anti-IgE antibody in adults and children with severe asthma? (Chosen biomarkers being $F_{\mathrm{ENO}}$, peripheral or sputum eosinophils and serum periostin)

4 Should a long-acting inhaled muscarinic antagonist be used in adults and children with severe asthma?

5 Should a macrolide (i.e. azithromycin, clarithromycin) be used in adults and children with severe asthma?

6 Should a monoclonal anti-IL-4R $\alpha$ be used in adults and children with severe asthma?

IL: interleukin; R: receptor; $F_{E N O}$ : exhaled nitric oxide fraction. ${ }^{\#}$ : for the purposes of this guideline, age $>5$ years.

\section{Group composition}

The ERS and ATS selected the Task Force co-chairs (Fernando Holguin and Andy Bush), who led the project and selected the other panellists, who included 23 clinicians and researchers with experience in severe asthma and two severe asthma patient representatives (Betty Frankemölle and Dominique Hamerlijnck). Two methodologists (David Rigau and Rebecca L. Morgan), led by the ERS senior methodologist (Thomy Tonia), supervised and ensured that all the methodological requirements were met. Systematic reviews and application of the GRADE approach were performed by members of the Task Force (Diogenes S. Ferreira and Sarah Diver) and externally commissioned (Iberoamerican Cochrane Centre, Barcelona, Spain). The methodologists took part in the Task Force meetings, but did not participate in the formulation of recommendations and had no voting rights.

The co-chairs and panellists discussed the evidence and formulated the recommendations. Evidence profiles and Evidence to Decision tables (supplementary material) developed with the GRADEpro Guideline Development Tool (gradepro.org) were used to facilitate the discussion, which was followed by voting on the recommendations. All panel members disclosed their conflicts of interest. Both co-chairs were required to be free from conflicts of interest relating to the management of asthma. Individuals with relevant conflicts of interest took part in the discussions about the evidence, but did not participate in the formulation of recommendations related to the questions where they had a relevant conflict of interest.

Thresholds for minimum clinically important differences (MCIDs) between treatment groups primarily in adults (used to judge imprecision according to GRADE) included the following absolute reductions: St George's Respiratory Questionnaire (SGRQ) score change of 4 points, Asthma Control Questionnaire (ACQ-5, ACQ-6 and ACQ-7) score change of 0.5 points, Asthma Quality of Life Questionnaire (AQLQ) score change of 0.5 points and forced expiratory volume in $1 \mathrm{~s}\left(\mathrm{FEV}_{1}\right)$ change of $0.23 \mathrm{~L}(10.38 \%$ change from baseline) [5-7].

\section{Literature searches}

The librarians (Shandra Knight and Liz Kellermeyer) conducted the literature search strategies in MEDLINE In-Process \& Other Non-Indexed Citations, MEDLINE, Embase and Cochrane Central Register of Controlled Trials, beginning in 2008 and ending with a final update on September 27, 2018. These dates were selected to capture developments in severe asthma therapy since the previous ERS/ATS guidelines. The literature searches included systematic reviews of randomised clinical trials including (moderate to severe) asthma populations receiving the interventions of interest. We excluded phase 1 (pharmacokinetic or pharmacodynamic studies), real-life nonrandomised extension studies and research reported in abstract form only (e.g. poster or congress presentations).

Results were limited to human subjects and to reports in the English language. Each strategy incorporated medical subject headings and text words for the topic of asthma, with search hedges for specific concepts defined in the PICOs. To supplement the electronic search, experts were contacted and reference lists were hand-searched.

\section{Evidence synthesis}

Study characteristics, types of participants, interventions, outcome measures and results were extracted from each study. If the data were amenable to pooling, effects were estimated by meta-analysis using 
Review Manager version 5.3 (The Nordic Cochrane Centre, Copenhagen, Denmark). For the meta-analyses, the random effects model was utilised unless otherwise specified. Dichotomous outcomes were reported as relative risks and continuous outcomes were reported as mean differences, unless otherwise specified. Absolute differences are reported in the accompanying documents in the supplementary material. Judgements on the quality of evidence were reviewed by the Task Force members and validated by the ERS methodologists (Thomy Tonia, David Rigau and Rebecca L. Morgan).

\section{Formulating and grading recommendation}

The evidence profiles were sent to the Task Force members for review. Using an iterative consensus process conducted face-to-face and also via teleconference and e-mail, and finally a vote by all members of the Task Force who had no relevant conflicts, recommendations were formulated on the basis of the following considerations: the balance of desirable (benefits) and undesirable consequences (burden, adverse effects and cost) of the intervention, the quality of evidence, patient values and preferences, and feasibility.

A strong recommendation was made for or against an intervention when the panel was certain that the desirable consequences outweighed the undesirable consequences (or the converse for a recommendation against). A strong recommendation is one that most well-informed patients would follow.

A conditional recommendation was made for or against an intervention when the panel was uncertain that the desirable consequences of the intervention outweighed the undesirable consequences (or the converse for a recommendation against). Reasons for uncertainty included low or very low quality of evidence, the desirable and undesirable consequences being finely balanced, the population in reviewed studies not uniformly meeting ERS/ATS severe asthma criteria, or the underlying values and preferences playing an important role. A conditional recommendation indicates that well-informed patients may make different choices regarding whether to have or not have the intervention.

\section{Manuscript preparation}

The two co-chairs, ERS methodologists and one panellist (Kian Fan Chung) developed the initial manuscript draft. The ERS methodologists and PICO leaders prepared the Evidence to Decision tables in the supplementary material. All materials were edited and approved by all panel members.

Supporting documentation, including GRADE Evidence profiles and the Evidence to Decision Frameworks tables, is included in the supplementary material.

\section{Results}

\section{Question 1: Should a monoclonal anti-IL-5 antibody be used in adults and children with severe} asthma?

IL-5 is a principal cytokine driving eosinophilic inflammation in asthma. Monoclonal antibodies that target IL-5 (mepolizumab, reslizumab) or its receptor IL-5R $\alpha$ (benralizumab) have been found to be efficacious in randomised controlled trials in improving asthma-related outcomes and are currently approved by the US Federal Drug Administration (FDA)/European Medicines Agency (EMA). We identified 12 randomised controlled trials that met inclusion criteria [8-19]. We included data only for participants on FDA/EMA licensed doses or the $20 \mathrm{mg}$ subcutaneous dose from phase 2 benralizumab trials. The evidence from meta-analyses of these trials is summarised in the following section. Asthma exacerbations, symptoms, asthma control, quality of life, use of systemic corticosteroids and adverse events were considered "critical outcomes". Change in lung function was deemed an "important" outcome.

\section{Summary of the evidence \\ Mepolizumab}

Three studies in adolescents and adults met inclusion criteria [8-10]. All three were randomised placebo-controlled trials in patients with severe eosinophilic asthma (blood eosinophil count $\geqslant 300 \mu \mathrm{L}^{-1}$ in the 12 months prior to screening or $\geqslant 150 \mu \mathrm{L}^{-1}$ during screening/oral corticosteroid (OCS) optimisation period) considered by this Task Force to represent a population of severe asthma subjects as defined by the ERS/ATS guidelines on severe asthma. Two studies required patients to have had at least two exacerbations in the previous year despite regular use of high-dose inhaled corticosteroid (ICS) plus another controller $[9,10]$, whereas the other study investigated the steroid-sparing effect of mepolizumab in OCS-dependent asthma [8].

Mepolizumab therapy was associated with a $50 \%$ reduction in the rate of any exacerbation (rate ratio 0.5 , 95\% CI 0.39-0.65; absolute risk 0.92 versus 1.69 events.patient ${ }^{-1} \cdot$ year $^{-1}$ ) and $64 \%$ reduction in exacerbations requiring an emergency department visit or hospitalisation (rate ratio 0.36, 95\% CI 0.20 0.66 ; absolute risk 0.05 versus 0.15 events.patient ${ }^{-1} \cdot$ year $^{-1}$ ). Compared with placebo, those assigned to 
mepolizumab experienced an absolute 0.43-point decrease (i.e. improvement) in ACQ-5 (95\% CI -0.56-0.31 points) and an absolute 7.14-point decrease (i.e. improvement) in SGRQ (95\% CI -9.07-5.21 points). Mepolizumab, relative to placebo, resulted in a 50\% median (95\% CI 20-75\%) reduction in the dose of maintenance OCS in one study of 135 patients [8]. The effect of mepolizumab on $\mathrm{FEV}_{1}$ was less than the MCID threshold.

\section{Reslizumab}

Four publications that included five randomised controlled trials met the inclusion criteria [11-14]. CASTRO et al. [13] reported on two duplicate trials. Three of the five randomised controlled trials included adolescents in addition to adult participants [11, 13]. All studies except one [12] included patients with mixed severity (moderate and severe) asthma. Three randomised controlled trials used inclusion criteria of blood eosinophils $\geqslant 400 \mu \mathrm{L}^{-1}[11,13,14]$ and one randomised controlled trial used sputum eosinophils $\geqslant 3 \%$ [12]. One randomised controlled trial included participants unselected for blood eosinophil count, but subsequently performed a subgroup analysis using a blood eosinophil cut-off of $400 \mu \mathrm{L}^{-1}$ [14]. Overall, reslizumab therapy was associated with a 54\% reduction in any exacerbation (rate ratio $0.46,95 \%$ CI $0.37-0.58$; absolute risk 0.84 versus 1.81 events.patient ${ }^{-1} \cdot$ year $^{-1}$ ) relative to placebo and $33 \%$ reduction in exacerbations requiring emergency department visits or hospitalisations (rate ratio 0.67, 95\% CI 0.391.17; absolute risk 0.077 versus 0.12 events.patient ${ }^{-1} \cdot$ year $^{-1}$ ). Reslizumab therapy also reduced the risk of patients having at least one exacerbation ( $29.2 \%$ versus $46.7 \%$; risk ratio 0.63 , 95\% CI $0.53-0.76$ ). In study participants meeting ERS/ATS criteria for diagnosis of severe asthma, reslizumab therapy was associated with a $60 \%$ reduction in the risk of having one or more exacerbations $(7.5 \%$ versus $18.9 \%$; risk ratio 0.40 , 95\% CI 0.13-1.20)

Relative to participants on placebo, those assigned to reslizumab experienced an absolute 0.26-point decrease (i.e. improvement) in ACQ-7 (95\% CI $-0.33--0.18$ points) and an absolute 0.28-point increase (i.e. improvement) in AQLQ (95\% CI $0.17-0.39$ points). The effect of reslizumab on $\mathrm{FEV}_{1}$ did not cross the MCID threshold.

\section{Benralizumab}

Five randomised controlled trials evaluating benralizumab met the inclusion criteria [15-19]. Four studies included a mixed population of patients with moderate or severe asthma [15-18]. Two of the five randomised controlled trials included adolescents in addition to adult participants [15, 17]. One study investigated the steroid-sparing effect of benralizumab in OCS-dependent asthma [18].

Overall, benralizumab therapy was associated with a $42 \%$ reduction in the rate of any exacerbation (rate ratio $0.58,95 \%$ CI $0.47-0.73$; absolute risk 0.64 versus 1.19 events.patient $^{-1} \cdot$ year $^{-1}$ ) and a $38 \%$ reduction in the number of patients with one or more exacerbations (35.9\% versus $51.1 \%$; risk ratio 0.62 , 95\% CI 0.36 1.06) relative to placebo. In study participants meeting ERS/ATS criteria for diagnosis of severe asthma, benralizumab therapy was associated with $55 \%$ reduction in exacerbations (number of patients with one or more exacerbations $23.3 \%$ versus $52 \%$; risk ratio 0.45 , 95\% CI $0.28-0.72$ ). Those requiring emergency department visits or hospitalisations were also reduced (rate ratio 0.45, 95\% CI 0.14-1.47; absolute risk 0.043 versus 0.18 events.patient ${ }^{-1} \cdot$ year $^{-1}$ ) and with a greater magnitude for patients meeting ERS/ATS criteria for diagnosis of severe asthma (rate ratio $0.07,95 \%$ CI $0.01-0.63$; absolute risk 0.02 versus 0.32 events.patient ${ }^{-1} \cdot$ year $^{-1}$ )

Relative to participants on placebo, those assigned to benralizumab experienced an absolute 0.29-point decrease (i.e. improvement) in ACQ-6 (95\% CI -0.4--0.17 points) and an absolute 0.32-point increase (i.e. improvement) in AQLQ (95\% CI 0.19-0.45 points). The effect of benralizumab on $\mathrm{FEV}_{1}$ was below the MCID. The median OCS dose reduction from baseline (range) at the final visit (week 28) was $25.0 \%$ $(-150-100 \%)$ in the placebo group $(n=75)$ and $75.0 \%(-50-100 \%)$ in the benralizumab group $(n=73)$ [18].

\section{Adverse effects}

Compared with placebo, the risk ratio of developing any adverse event for a participant was 0.93 (95\% CI $0.88-0.99)$ for mepolizumab (74.8\% versus $79.6 \%), 0.88$ (95\% CI $0.81-0.96)$ for reslizumab (67.1\% versus $80.4 \%)$ and 0.96 (95\% CI $0.91-1.01)$ for benralizumab (73.6\% versus $75.5 \%)$. Similarly, participants experienced a lower risk of serious adverse events when assigned to anti-IL-5 strategy drugs (supplementary material). The lower risk for having any adverse events is likely driven by the reduction in severe asthma exacerbations by these drugs.

Data are available on drug-related adverse events from all three mepolizumab trials, but only from two out of five reslizumab trials and one out of five benralizumab trials. These data show that, relative to placebo, participants assigned to mepolizumab had a greater risk of drug-related adverse events (13.3\% versus $9.2 \%$; risk ratio $1.35,95 \%$ CI 1.01-1.80), those assigned to reslizumab had a lower risk ( $8 \%$ versus $11.9 \%$; risk 
ratio $0.69,95 \%$ CI $0.44-1.09)$ and those assigned to benralizumab had a greater risk (13.3\% versus $9.2 \%$; risk ratio $1.46,95 \% \mathrm{CI} 0.96-2.21$ ). As the outcome drug-related adverse events were not pre-defined, the Task Force members did not consider this outcome in the overall certainty of the evidence of effects.

\section{Benefits}

Anti-IL-5 and anti-IL-5R $\alpha$ therapies reduce exacerbations and hospitalisations in patients with severe eosinophilic asthma. Mepolizumab and benralizumab are effective in reducing maintenance OCS dose in patients with corticosteroid-dependent severe asthma.

\section{Harms}

All three anti-IL-5 strategy drugs were well tolerated. The frequency of adverse effects was similar when compared with placebo.

\section{Conclusions}

An anti-IL-5 strategy reduces exacerbations in patients with severe eosinophilic asthma. Mepolizumab and benralizumab are effective in reducing OCS dose in corticosteroid-dependent asthma. The effects on asthma control, quality of life and $\mathrm{FEV}_{1}$ are modest for all drugs and did not meet the MCID threshold.

\section{Research needs and additional considerations}

Direct comparisons will be needed to further guide selection of the appropriate anti-IL-5 drug. Uncertainty exists around the best biomarker and blood eosinophil threshold that would predict response to anti-IL-5 therapy. In addition to blood eosinophils, the efficacy of anti-IL-5 therapy depends on the degree of pre-existing asthma exacerbations. This should be taken into consideration when considering the clinical and cost-effectiveness of this form of therapy. Data from adolescents are unavailable for mepolizumab and reslizumab, whereas for benralizumab there are data on a limited number of adolescents with severe asthma. There are no data on younger children (age $<5$ years). Therefore, more evidence is needed to provide higher quality recommendations in the paediatric age group.

What others are saying

The 2019 Global Initiative for Asthma (GINA) guidelines [20] and the National Institute for Health and Care Excellence (NICE) technology appraisal guidance [21-23] include mepolizumab, reslizumab and benralizumab as add-on therapeutic options for severe eosinophilic asthma (at GINA step 5).

\section{ERS/ATS recommendation}

We suggest an anti-IL-5 strategy as add-on therapy for adult patients with severe uncontrolled asthma with an eosinophilic phenotype and for those with severe corticosteroid-dependent asthma (the Task Force gave this a conditional recommendation because inclusion criteria across studies did not consistently align with the ERS/ATS severe asthma definition).

\section{Remarks}

The high cost of these drugs and its impact on cost-effectiveness, equity and feasibility to implementation must be weighed by clinicians in relation to the benefits on asthma outcomes shown by all anti-IL- 5 and anti-IL-5R $\alpha$ strategy drugs [24]. Due to the limited number of treated adolescents or children, the Task Force was unable to provide a recommendation for the use of anti-IL-5 and anti-IL-5R $\alpha$ antibodies in this age group.

\section{Question 2: Should a measurement of a specific biomarker be used to guide initiation of treatment with a monoclonal anti-IL-5 or IL-5R $\alpha$ antibody in adults and children with severe asthma? (Chosen biomarkers being $\mathrm{F}_{E N O}$, peripheral or sputum eosinophils and serum periostin) Summary of the evidence}

We identified 12 randomised controlled trials of anti-IL-5 therapies in children and adults 12-75 years of age that evaluated differential response to therapy among subgroups of individuals with higher or lower levels of eosinophils in blood or sputum in post hoc analyses [10-17, 19, 25, 26]. One study was a meta-analysis of two randomised controlled trials of mepolizumab's therapeutic responsiveness combining the $100 \mathrm{mg}$ s.c. and $75 \mathrm{mg}$ intravenous doses for the analysis by blood eosinophil level [26]. Notably, four of the studies recruited only subjects with evidence of eosinophilic asthma, defined as sputum eosinophils $\geqslant 3 \%$ or blood eosinophil level $\geqslant 300 \mu \mathrm{L}^{-1}[11-13,25]$. Six of the studies included children age $\geqslant 12$ years $[10,11,13,15,17,26]$. The most commonly measured biomarker was blood eosinophil count. Only one study evaluated sputum eosinophil level [12]. One additional study evaluated whether the presence of persistently elevated sputum or blood eosinophils was an indicator of therapeutic failure and justified the addition of an alternate anti-IL-5 strategy [27]. 
Cut-offs assessed for baseline blood eosinophil levels, and hence the definition of what constitutes eosinophilia, varied across anti-IL-5 strategies. Studies of mepolizumab specifically assessed a cut-off of blood eosinophils $\geqslant 150 \mu \mathrm{L}^{-1}$. For mepolizumab, there was a $73 \%$ (95\% CI $-82--59 \%$ ) reduction in exacerbations among those with a blood eosinophil level $\geqslant 500 \mu \mathrm{L}^{-1}$ compared with a $36-39 \%$ reduction in all other groups with eosinophil levels $\geqslant 150 \mu \mathrm{L}^{-1}$. Notably, subjects with eosinophil levels $\geqslant 150 \mu \mathrm{L}^{-1}$ constituted nearly three-quarters of the severe asthma population in those studies. Patients treated with reslizumab with a baseline eosinophil level $\geqslant 400 \mu \mathrm{L}^{-1}$ had a $54 \%$ reduction in exacerbations; higher cut-offs were not associated with a greater reduction in exacerbations. For benralizumab, a cut-off $\geqslant 300 \mu \mathrm{L}^{-1}$ was associated with a significant reduction in exacerbations; however, it is not clear what the optimal cut-off should be since even subjects with an eosinophil level $<300 \mu \mathrm{L}^{-1}$ experienced a reduction in exacerbations.

For effects on asthma control and quality of life, the data again varied by anti-IL-5 strategy; among those with a baseline eosinophil level $\geqslant 150 \mu \mathrm{L}^{-1}$, 63\% treated with mepolizumab versus $41 \%$ treated with placebo achieved $a \geqslant 0.5$-point reduction from baseline in ACQ-5 (risk ratio 1.53, 95\% CI 1.27-1.84). The improvement in asthma control was similar among those with higher baseline levels of eosinophils $(\geqslant 300$ or $\left.\geqslant 500 \mu \mathrm{L}^{-1}\right)$. For benralizumab, only subjects with a baseline eosinophil level $\geqslant 300 \mu \mathrm{L}^{-1}$ experienced a significant improvement in asthma control, assessed as change in ACQ-6 score from baseline (mean difference -0.28 points, $95 \%$ CI $-0.41--0.15$ points), whereas those with an eosinophil level $<300 \mu \mathrm{L}^{-1}$ did not (mean difference -0.20 points, $95 \%$ CI $-0.44-0.3$ points). Similarly for reslizumab, a cut-off eosinophil level $\geqslant 400 \mu \mathrm{L}^{-1}$ was associated with improved asthma control (mean difference in ACQ-7) from baseline -0.27 points (95\% CI $-0.36--0.19$ points), whereas those with an eosinophil level $<400 \mu \mathrm{L}^{-1}$ did not have a significant benefit $(-0.12$ points, $95 \% \mathrm{CI}-0.33-0.09$ points). Sputum eosinophil level was only considered in one study of reslizumab [12] and sputum levels were categorised as $\geqslant 10 \%$ or $<10 \%$. There were no statistical differences found between groups in level of asthma control. There was a trend for higher blood eosinophil levels to be associated with a greater improvement in asthma control.

One additional study, which was not included in the meta-analysis, assessed treatment response of weight-adjusted i.v. reslizumab in patients previously treated with $100 \mathrm{mg}$ s.c. mepolizumab [27]. It reported that persistently high levels of eosinophils (blood $>300 \mu \mathrm{L}^{-1}$ and sputum $>3 \%$ ) after treatment with mepolizumab characterised responders. In those subjects a weight-adjusted dose of reslizumab was administered. It was found that further improvements in symptoms and reductions in eosinophilia were possible with addition of reslizumab. These data suggest that evidence of uncontrolled eosinophilic inflammation, as manifest by a high sputum or blood eosinophil level, may be useful in determining which subjects may benefit from additional anti-IL-5 strategies; however, this requires further confirmation.

\section{Benefits}

The specific cut-off blood eosinophil count to predict improved asthma control and reduction in exacerbations varies across anti-IL-5 strategies. However, there is very low quality evidence that mepolizumab may provide further benefit in reducing exacerbations in patients with baseline blood eosinophilia $\geqslant 500 \mu \mathrm{L}^{-1}$ compared with those with eosinophil levels $<150,150-<300$ and $300-<500 \mu \mathrm{L}^{-1}$.

\section{Harms}

There were five studies that assessed adverse events in benralizumab or reslizumab [11, 13-17]. The data for mepolizumab did not assess differences in adverse event rates based on blood eosinophil level. There was no difference in adverse events among those with higher versus lower eosinophil counts for benralizumab. For reslizumab, only subjects with baseline eosinophilia $\geqslant 400 \mu \mathrm{L}^{-1}$ during screening were recruited; the fewest adverse events occurred in the group who had no data on eosinophil count at the time of recruitment compared with patients with baseline eosinophilia $\geqslant 400 \mu \mathrm{L}^{-1}$. There was a $5 \%$ reduction in the number of adverse events among those with an eosinophil count $\geqslant 400 \mu \mathrm{L}^{-1}$ which, although statistically relevant, may not be clinically meaningful. More recent studies have now shown that both benralizumab and mepolizumab maintain an adequate safety profile during long-term use for up to 2 and 4.5 years, respectively $[28,29]$.

\section{Other considerations}

Most of the studies focused on blood eosinophils as a biomarker, and there were limited data on sputum eosinophils and no data on $F_{\mathrm{ENO}}$ or serum periostin. Blood eosinophils can be measured in any standard laboratory, increasing its feasibility as a biomarker; however, additional testing beyond the point of care may be required to ascertain baseline levels, particularly among patients on or recently taking systemic corticosteroids. It is more acceptable than sputum eosinophil levels, which are currently only performed in 
specialised centres. It should be noted that there may be causes other than atopy (e.g. parasitic infections) for peripheral blood eosinophilia, especially in low- and middle-income settings.

Cut-offs to assess response varied across studies of anti-IL-5 medications and there were no data comparing therapeutic regimens using different cut-off levels. Finally, most of the anti-IL-5 strategies use a fixed-dose regimen based on randomised controlled trial data suggesting a plateau in the dose response; however, one study suggested that persistent eosinophilia, despite anti-IL-5 strategies, should be considered as an opportunity to add-on reslizumab using a weight-adjusted dose regimen [27].

\section{Conclusions and research needs}

Although the data suggest that subjects with higher levels of blood eosinophil counts benefit more from anti-IL-5 strategies, the evidence we reviewed does not show that a specific level of blood eosinophils $\geqslant 150 \mu \mathrm{L}^{-1}$ for mepolizumab, $\geqslant 300 \mu \mathrm{L}^{-1}$ for benralizumab and $\geqslant 400 \mu \mathrm{L}^{-1}$ for reslizumab is an absolute response threshold, as clinical benefit can still be observed in some patients below these values. Based on currently available evidence (which is very limited), sputum eosinophils may not add to the prediction of response greater than blood eosinophil level.

Determining a patient's baseline eosinophil count may require more than one measurement, as this biomarker is highly variable and significantly reduced by systemic corticosteroid and ICS. It is not known if eosinophil levels obtained during periods of asthma exacerbation are better predictors of treatment response when compared with those measured during periods of clinical stability. Future studies should focus on developing additional noninvasive biomarkers for adults and children that can be used at point of care to predict responsiveness to different anti-IL-5 strategies.

What others are saying

The 2019 GINA guidelines [20] recommend the use of an anti-IL-5 and anti-IL-5R $\alpha$ strategy for patients who who remain uncontrolled despite step 4 or 5 therapy who have blood eosinophils $\geqslant 300 \mu \mathrm{L}^{-1}$.

\section{ERS/ATS recommendation}

We suggest that a blood eosinophil count cut-off point $\geqslant 150 \mu \mathrm{L}^{-1}$ can be used to guide anti-IL-5 initiation in adult patients with severe asthma and a history of prior asthma exacerbations (conditional recommendation, low quality evidence).

\section{Remarks}

The Task Force placed a high value on reducing exacerbations and a greater feasibility of biomarker measurement, and a lower value on cost and invasiveness.

Question 3: Should a measurement of a specific biomarker be used, in addition to total IgE level, to guide initiation of treatment with a monoclonal anti-lgE antibody in adults and children with severe asthma? (Chosen biomarkers being $\mathrm{F}_{E N O}$, peripheral or sputum eosinophils and serum periostin)

Summary of the evidence

We identified three randomised, double-blind placebo-controlled trials [31-33] which recruited participants age 12-75 years. Of these, two studies [32,33] involving 1014 eligible participants formed the evidence for the Task Force recommendations. These two trials included individuals with uncontrolled asthma. In one study [33], patients had uncontrolled symptoms while taking an ICS with or without a controller. In the other study [32], only participants with severe persistent asthma were recruited, whose asthma remained uncontrolled despite ICS and a long-acting $\beta_{2}$-agonist (LABA).

In both trials, eligible participants were randomised 1:1 to receive omalizumab or placebo. Omalizumab dose was determined on the basis of pre-treatment serum total IgE level $\left(\mathrm{IU} \cdot \mathrm{mL}^{-1}\right)$ and body weight $(\mathrm{kg})$ according to the European [33] or ATS [32] omalizumab dosing table, which ensured a minimum omalizumab dose of $0.008 \mathrm{mg} \cdot \mathrm{kg}^{-1} \cdot \mathrm{IgE}^{-1}\left(\mathrm{IU} \cdot \mathrm{mL}^{-1}\right)$ every 2 weeks or a minimum of $0.016 \mathrm{mg} \cdot \mathrm{kg}^{-1} \cdot \mathrm{IgE}^{-1}$ $\left(\mathrm{IU} \cdot \mathrm{mL}^{-1}\right)$ every 4 weeks.

Busse et al. [33] pre-planned an analysis that divided participants into two subgroups according to eosinophil counts at screening: low $\left(<300 \mu \mathrm{L}^{-1}\right)$ and high $\left(\geqslant 300 \mu \mathrm{L}^{-1}\right)$. A subgroup analysis was performed by HaNANIA et al. [32], which divided participants into high and low subgroups as: $F_{\mathrm{ENO}}$ low $<19.5 \mathrm{ppb}$, high $\geqslant 19.5 \mathrm{ppb}$; peripheral blood eosinophils low $<260 \mu \mathrm{L}^{-1}$, high $\geqslant 260 \mu \mathrm{L}^{-1}$; and serum periostin levels low $<50 \mathrm{ng} \cdot \mathrm{mL}^{-1}$, high $\geqslant 50 \mathrm{ng} \cdot \mathrm{mL}^{-1}$.

Pooling of the data from the two studies was not possible. In Busse et al. [33], there were significant improvements in exacerbation rates (hazard ratio $0.41,95 \%$ CI $0.20-0.84$ ) and a clinically trivial but 
statistically significantly greater change in $\mathrm{FEV}_{1} \%$ pred at 24 weeks (mean difference 7.35 , 95\% CI 1.38 13.31) with omalizumab compared with placebo in patients with a high eosinophil count, whereas there were no differences in patients with low eosinophils $\left(<300 \mu \mathrm{L}^{-1}\right)$. In the study by Hanania et al. [32], there was a significantly longer time to first asthma exacerbation with omalizumab compared with placebo in patients with high $\left(\geqslant 260 \mu \mathrm{L}^{-1}\right)$ eosinophil count at 48 weeks follow-up (hazard ratio $0.64,95 \%$ CI 0.48 . $0.85)$, whereas there were no differences in patients with low $\left(<260 \mu \mathrm{L}^{-1}\right)$ eosinophil count (hazard ratio 0.95, 95\% CI 0.68-1.33). However, there were no statistically significant differences between these subgroups. There were no differences in AQLQ at 48 weeks when omalizumab was compared with placebo in patients with high $\left(\geqslant 260 \mu \mathrm{L}^{-1}\right)$ eosinophils (mean difference 0.14 points, $95 \% \mathrm{CI}-0.11-0.30$ points), while there was a small statistically, but not clinically significant, difference in the low $\left(<260 \mu \mathrm{L}^{-1}\right)$ eosinophil subgroup (mean difference 0.26 points, 95\% CI 0.06-0.46 points).

In the subgroup analysis by $F_{\mathrm{ENO}}$ [32], there was a significant relative reduction of exacerbation rates with omalizumab compared with placebo in patients with high $(\geqslant 19.5 \mathrm{ppb}) F_{\mathrm{ENO}}$ at 48 weeks follow-up (53\%, 95\% CI $37-70 \%)$, whereas there were no differences for those patients with low $(<19.5 \mathrm{ppb}) F_{\mathrm{ENO}}(16 \%$, 95\% CI $-32-46 \%)$. The time to first asthma exacerbation with omalizumab, compared with placebo, was significantly longer in patients with high $(\geqslant 19.5 \mathrm{ppb}) F_{\mathrm{ENO}}$ at 48 weeks follow-up (hazard ratio $0.38,95 \%$ CI 0.24-0.60), whereas there were no differences in patients with low ( $<19.5 \mathrm{ppb}) F_{\text {ENO }}$ (hazard ratio 1.00 , 95\% CI 0.62-1.61). There were also larger changes of mean AQLQ with omalizumab compared with placebo in high $(\geqslant 19.5 \mathrm{ppb}) F_{\text {ENO }}$ patients at 48 weeks of follow-up (mean difference 0.39 points, $95 \%$ CI 0.06-0.72 points), whereas there were no differences in low ( $<19.5 \mathrm{ppb}) F_{\mathrm{ENO}}$ patients (mean difference 0.24 points, $95 \%$ CI $-0.09-0.57$ points).

There were no differences in the relative reduction of exacerbation rates at 48 weeks or $\mathrm{FEV}_{1}$ when omalizumab was compared with placebo in periostin high $\left(\geqslant 50 \mathrm{ng} \cdot \mathrm{mL}^{-1}\right)$ or low $\left(<50 \mathrm{ng} \cdot \mathrm{mL}^{-1}\right)$ patients [32]. However, compared with placebo, omalizumab improved AQLQ in patients with low $\left(<50 \mathrm{mg} \cdot \mathrm{mL}^{-1}\right)$ periostin levels at 48 weeks follow-up (mean difference 0.50 points, $95 \%$ CI $0.22-0.78$ points), whereas there were no differences patients with high $\left(\geqslant 50 \mathrm{ng} \cdot \mathrm{mL}^{-1}\right)$ serum periostin levels (mean difference 0.10 points, $95 \%$ CI $-0.19-0.39$ points).

\section{Benefits}

In patients treated with omalizumab compared with placebo, the presence of a baseline blood eosinophil count $\geqslant 260 \mu \mathrm{L}^{-1}$ is associated with greater improvements in $\mathrm{FEV}_{1}$, and a decreased rate of exacerbations as well as longer time to first exacerbation, compared with those with a blood eosinophil count $<260 \mu \mathrm{L}^{-1}$.

In patients treated with omalizumab compared with placebo, the presence of $F_{\mathrm{ENO}} \geqslant 19.5 \mathrm{ppb}$ is associated with improvements in AQLQ, reduced exacerbation rate and longer time to first exacerbation compared with those with $F_{\mathrm{ENO}}<19.5 \mathrm{ppb}$. In patients treated with omalizumab compared with placebo, the presence of periostin $<50 \mathrm{ng} \cdot \mathrm{mL}^{-1}$ was associated with improvements in AQLQ compared with those with periostin $\geqslant 50 \mathrm{ng} \cdot \mathrm{mL}^{-1}$. Periostin levels, however, did not predict response in exacerbations or lung function. There is no evidence that periostin is a suitable biomarker to guide asthma treatment in children or adolescents. Levels are influenced by age, skeletal growth and puberty [34].

\section{Harms}

There were no differences in the adverse effects in patients treated with omalizumab versus placebo according to high or low $F_{\mathrm{ENO}}$, blood eosinophils or periostin.

\section{Other considerations}

The estimates of effect included one single study (meta-analysis of the two randomised controlled trial was not possible), which introduced some uncertainty due to the limited number of patients included in each subgroup according to the biomarker threshold. Furthermore, the risk of bias was high for completeness of data, due to a considerable number of patients that were not evaluated at baseline for the biomarkers.

\section{Conclusions and research needs}

Blood eosinophil counts and $F_{\mathrm{ENO}}$ levels may be useful in choosing patients most likely to achieve a more positive effect on exacerbations and lung function when treated with omalizumab compared with placebo. There were no differences in adverse effects based on the high and low biomarker subgroups, suggesting that the high blood eosinophil and high $F_{\mathrm{ENO}}$ patients achieve clinical benefit without additional adverse effects, whereas low biomarker patients are at risk of adverse effects while potentially having less clinical benefit.

Other excluded studies also make important observations regarding the use of blood eosinophils to select patients most likely to respond to omalizumab. Of particular note is the study by CASALE et al. [35], who 
reported an analysis that pooled the results of two randomised controlled trials. The studies by Busse et al. [36] and Soler et al. [37] were both phase 3, double-blind placebo-controlled trials, comprising a total of 1071 participants comparing omalizumab with placebo in participants with moderate to severe asthma. The pooled analysis published in 2018 investigated the annualised exacerbation rates in the omalizumab group versus placebo according to the subgroups of blood eosinophils: high $\left(\geqslant 300 \mu \mathrm{L}^{-1}\right)$ and low $\left(<300 \mu \mathrm{L}^{-1}\right)$ [35]. The results support the recommendations of the Task Force. There was a more pronounced reduction in exacerbations rates in the omalizumab versus placebo group for the high biomarker subgroup, i.e. for those with an eosinophil count $\geqslant 300 \mu \mathrm{L}^{-1}$ there was a $67 \%$ reduction in exacerbations compared with a $45 \%$ reduction in the $<300 \mu \mathrm{L}^{-1}$ group.

In contrast to the previous studies, one publication found that omalizumab's effectiveness did not vary across biomarker levels. This retrospective study of 872 patients with severe allergic asthma showed that omalizumab reduced exacerbations by $58.4 \%$ (95\% CI 52.7-63.4\%) in the high biomarker (eosinophil count $\geqslant 300 \mu \mathrm{L}^{-1}$ ) group versus $58.1 \%$ (95\% CI 52.7-63.4\%) in the low biomarker group (eosinophil count $<300 \mu \mathrm{L}^{-1}$ ) [38].

Future randomised controlled trials should evaluate baseline blood eosinophils and $F_{\text {ENO }}$ as individual and combined biomarkers to further determine their ability to predict response to treatment for multiple outcomes including exacerbations, lung function as well as patient-reported outcomes such as AQLQ and asthma control. Furthermore, there is a need to identify biomarkers that support clinical decision making regarding the continuation versus discontinuation of a monoclonal anti-IgE strategy in adults and children with severe asthma.

What others are saying

The 2019 GINA guidelines [20] state that blood eosinophil level $\geqslant 260 \mu \mathrm{L}^{-1}$ and $F_{\mathrm{ENO}} \geqslant 20 \mathrm{ppb}$ are factors that may predict a good response to treatment.

Neither the 2019 British Thoracic Society/Scottish Intercollegiate Guidelines Network (BTS/SIGN) [39] nor the NICE asthma guidelines [30] make comment about predictor biomarkers for anti-IgE treatment response.

\section{ERS/ATS recommendations}

In adult and adolescent patients with severe asthma being considered for omalizumab we suggest:

- Using a blood eosinophil cut-off $\geqslant 260 \mu \mathrm{L}^{-1}$ to identify adolescents (>12 years) and adults with severe allergic asthma more likely to benefit from anti-IgE treatment (conditional recommendation, low quality of evidence).

- Using a $F_{\mathrm{ENO}}$ cut-off $\geqslant 19.5 \mathrm{ppb}$ to identify adolescents ( $>12$ years) and adults with severe allergic asthma more likely to benefit from anti-IgE treatment (conditional recommendation, low quality of evidence).

\section{Remarks}

Since these recommendations have not been prospectively evaluated, treatment decisions should consider these biomarker thresholds cautiously, as patients with eosinophil or $F_{\mathrm{ENO}}$ values below the proposed cut-offs can still benefit from omalizumab. In addition, these thresholds were largely determined by one particular study [32]. Periostin was omitted from these recommendations, as this biomarker is not clinically available and it is not useful in children age $<12$ years because it is also produced from growing bone.

The recommendations place a high value on an increased treatment response when blood eosinophils and $F_{\mathrm{ENO}}$ are used to select patients and a low value on the use of periostin.

\section{Question 4: Should a long-acting inhaled muscarinic antagonist be used in adults and children with severe asthma? \\ Summary of the evidence}

We identified three randomised, placebo-controlled trials in adults 18-75 years of age (one crossover and two parallel designs), one trial in adolescents (age 12-17 years) and one trial in children (age 6-11 years) [40-42]. These trials included individuals with severe uncontrolled asthma on GINA step 4-5 or NAEPP step 5 therapies. Adults were treated with at least high-dose ICS in combination with a LABA, while adolescents and children were treated with medium-dose ICS and a LABA with a third controller.

In the adolescent and paediatric studies, eligible patients were randomised in a 1:1:1 ratio to receive tiotropium $5 \mu \mathrm{g}$ ( 2 puffs of $2.5 \mu \mathrm{g}$ ) or $2.5 \mu \mathrm{g}$ ( 2 puffs of $1.25 \mu \mathrm{g}$ ) or placebo ( 2 puffs), each delivered for 
12 weeks via the Respimat Soft Mist inhaler as add-on to pre-enrolment background therapy with ICS plus one or more controller therapies. Whereas two adult studies [41] compared $5 \mu \mathrm{g}$ tiotropium (2 puffs of $2.5 \mu \mathrm{g}$ ) delivered by Respimat over 48 weeks to placebo, one adult study [40] involved an 8-week, three-way crossover design with $5 \mu \mathrm{g}$ tiotropium ( 2 puffs of $2.5 \mu \mathrm{g}$ ), $10 \mu \mathrm{g}$ tiotropium ( 2 puffs of $5 \mu \mathrm{g}$ ) and placebo, and was excluded from further analyses and the primary meta-analyses. The remaining four trials enrolled a total of 1433 participants $(2.5 \mu \mathrm{g}$ dose, $\mathrm{n}=528)$ and were pooled for meta-analyses to inform the Task Force's judgements.

Across the four parallel-arm trials including children, adolescents and adults, the addition of tiotropium $5 \mu \mathrm{g}$ resulted in improvements in mean peak $\mathrm{FEV}_{1}$ response compared with placebo (123 $\mathrm{mL}, 95 \% \mathrm{CI}$ 88.2-158.7 mL), which was statistically significant but a clinically trivial difference. Serious imprecision in the certainty estimates was also noted for each age group. The addition of tiotropium $5 \mu \mathrm{g}$ also marginally improved ACQ-7 ( -0.11 points, 95\% CI $-0.2-0.01$ points) and prevented asthma worsening (based on exacerbations or symptoms, risk ratio $0.79,95 \%$ CI $0.7-0.89$; absolute risk 133 , 95\% CI 54-122, fewer worsening episodes per 1000) compared with placebo, but again, serious imprecision in the certainty estimates was noted for children and adolescents. In children and adolescents, addition of tiotropium $2.5 \mu \mathrm{g}$ did not improve asthma control scores but did improve $\mathrm{FEV}_{1} \%$ pred (mean difference 4.99, 95\% CI 2.84-7.15) and reduced asthma worsening (risk ratio 0.66, 95\% CI 0.45-0.97). Post hoc analyses of adjusted mean trough $\mathrm{FEV}_{1}$ /forced vital capacity responses in children also demonstrated statistically significant improvements at all time-points versus placebo with both tiotropium doses, with the exception of tiotropium $2.5 \mathrm{mg}$ at week 8 .

In the two adult trials, treatment with tiotropium $5 \mu \mathrm{g}$ did not result in significant differences in AQLQ (mean difference 0.10 points, $95 \%$ CI $-0.04-0.23$ points), but did increase the time to first exacerbation requiring OCS (hazard ratio for placebo 0.79 , 95\% CI 0.62-1.01). Asthma exacerbations requiring hospitalisation were too infrequent in both the tiotropium (16 out of 453 subjects) and placebo (20 out of 454 subjects) arms to draw conclusions [41]. The crossover study in adults [40] that was excluded from the primary analysis similarly noted beneficial effects of tiotropium $5 \mu \mathrm{g}$ (mean difference $139 \mathrm{~mL}, 95 \%$ CI 96-181 mL) and $10 \mu \mathrm{g}$ (mean difference $170 \mathrm{~mL}, 95 \% \mathrm{CI} 128-213 \mathrm{~mL}$ ) on peak $\mathrm{FEV}_{1}$ response in adults.

Adverse events were less frequent in the tiotropium arms compared with placebo in these four trials (risk ratio $0.92,95 \%$ CI 0.86-0.98). Severe adverse events were equally infrequent across treatment arms.

\section{Benefits}

Long-acting muscarinic antagonist (LAMA) treatment in children, adolescents and adults with severe asthma may improve $\mathrm{FEV}_{1}$ and may reduce loss of asthma control. In adults, treatment with tiotropium $5 \mu \mathrm{g}$ also improves asthma control and increases time to first exacerbation.

\section{Harms}

There was a lower frequency of adverse events in children, adolescents and adults treated with tiotropium $5 \mu \mathrm{g}$ compared with placebo. The frequency of severe adverse events was also low and nearly equal to placebo.

\section{Conclusions and research needs}

The addition of tiotropium improves $\mathrm{FEV}_{1}$ and provides beneficial effects on symptom control in children, adolescents and adults with severe asthma not controlled with GINA step 4-5 and NAEPP step 5 combination therapies. There were too few severe exacerbations requiring OCS to draw definitive conclusions as to benefit. Based on the estimated beneficial effects observed for tiotropium, the Task Force judged that these benefits outweigh the adverse effects, burdens and costs associated with this treatment for the management of severe asthma.

In the combined age groups, tiotropium was effective in preventing the composite outcome for asthma worsening inclusive of symptom control and exacerbations. However, the effect of treatment was not significant in adolescents and children, likely due to the smaller sample sizes and shorter study duration of these trials. There is insufficient evidence for the beneficial effects of tiotropium on severe exacerbations in children and adolescents with severe asthma, which should be investigated in longer-term trial cohorts of sufficient size. There are additional LAMAs (umeclidinium and glycopyrronium) currently available which could be alternative long-term bronchodilator therapies for severe asthma. Treatments with umeclidinium and glycopyrronium have beneficial effects on lung function and symptom control in individuals with mild to moderate, persistent asthma [43-45], but have not been evaluated as an adjunct therapy for severe asthma. 
Future studies should also focus on the identification of severe asthma subgroups preferentially responsive to LAMAs that might benefit from the step-wide addition of muscarinic antagonists compared with alternative step-up options such as LABAs or increased ICS dosing. Subgroup analyses of trial cohorts with mild to moderate persistent asthma subjects have suggested that subgroups with fixed or baseline airflow obstruction might preferentially respond to LAMAs $[45,46]$. Three randomised controlled trials only included subjects with $\mathrm{FEV}_{1}<80 \%$ predicted. KERSTJENs et al. [47] showed beneficial effects in both those with screening $\mathrm{FEV}_{1}<60 \%$ or $60-80 \%$ predicted. Two trials in children and adolescents enrolled asthma patients with $\mathrm{FEV}_{1}$ 60-90\% predicted [42, 48]. Hence, it is not clear whether individuals, particularly adults, with severe asthma and higher lung function on combination therapy with high-dose ICS and a LABA will benefit from the addition of a LAMA.

A responder analysis of a severe asthma trial cohort showed equally beneficial effects when comparing subgroups based on baseline lung function, age, sex, ethnicity, body mass index and racial groups. Differential inter-racial effects are difficult to ascertain since minority racial groups (African-Americans and Asians) and Hispanic ethnic groups represented the vast minority of subjects in these trials [47]. Future trials in increasingly ethnically diverse severe asthma cohorts should provide insight into the beneficial effects of LAMAs in these groups, which experience a substantial proportion of asthma-related morbidity. Studies to evaluate responder subgroups based on genetic variation (pharmacogenetic studies) should also be performed using DNA samples from prior and future clinical trials.

What others are saying

The 2019 GINA guidelines [20] recommend the use of tiotropium as an add-on therapeutic option at step 4 or 5 for patients with exacerbations despite treatment with ICS and LABA.

The NAEPP guidelines [49] do not outline any role for the muscarinic antagonists.

\section{ERS/ATS recommendation}

For children, adolescents and adults with severe asthma uncontrolled despite GINA step 4-5 or NAEPP step 5 therapies, we recommend the addition of tiotropium (strong recommendation, moderate quality of evidence).

\section{Remarks}

While the Task Force only found data on the efficacy of $5 \mu \mathrm{g}$ in adults with severe asthma, the effects on lung function were similar to the FDA-approved 2.5 and $5 \mu \mathrm{g}$ doses evaluated in parallel, placebo-controlled trials of adults with mild to moderate asthma. In addition, clinical trials in adolescents with moderate and severe asthma showed that the 2.5 and $5 \mu \mathrm{g}$ doses were similarly effective. This recommendation places a high value on improving symptom control and reducing exacerbations. The strength of the recommendations is based on the following considerations when comparing the addition of tiotropium versus no addition. The evidence suggested with moderate certainty a large benefit and trivial harm, with the balance of effects clearly favouring the intervention. Tiotropium was considered probably acceptable and probably feasible to implement. This recommendation also accounts for the feasibility of this inhaled therapy compared with the cost and burden of alternative add-on biological therapies for severe asthma.

\section{Question 5: Should a macrolide (i.e. azithromycin, clarithromycin) be used in adults and children} with severe asthma?

Summary of the evidence

The previous ERS/ATS guidelines made a conditional recommendation that long-term macrolide antibiotics should not be used in the treatment of adults or children with severe asthma, based on available evidence [1]. Since then, six randomised controlled trials have been conducted [50-55], of which five included only adults and one included only children $6-<18$ years of age. There were varying definitions of persistent symptomatic or uncontrolled asthma and none met ERS/ATS criteria for severity. Three studies used azithromycin; of these, two (totalling 529 participants) used doses ranging from 250 to $500 \mathrm{mg}$ three times per week for a treatment period of $26-48$ weeks [50, 51]. The other $(n=97)$ used a dose of $600 \mathrm{mg} \cdot \mathrm{day}^{-1}$ for 3 days and $600 \mathrm{mg} \cdot \mathrm{week}^{-1}$ thereafter for 11 weeks [53]. The clarithromycin randomised controlled trials (totalling 171 participants) used $600 \mathrm{mg}$ twice daily, ranging from 8 to 16 weeks in treatment duration $[54,55]$. In children $(n=55)$, azithromycin nightly doses were given according to body weight, ranging from $250 \mathrm{mg}$ for $25-40 \mathrm{~kg}$ and $500 \mathrm{mg}$ for $>40 \mathrm{~kg}$ for a total of 12 months (the study was prematurely terminated at 30 weeks due to lack of clinical efficacy) [52].

Compared with placebo, during 48 weeks of follow-up, azithromycin reduced the number of combined moderate and severe exacerbations (1.07 versus 1.86 events.patient ${ }^{-1} \cdot$ year $^{-1}$; risk ratio 0.59 , 95\% CI $0.47-$ 
0.74) [51]. Additionally, macrolides reduced the number of patients with at least one moderate or severe asthma exacerbation and the time to first exacerbation. It did not, however, reduce the rate of severe exacerbations (25.3\% versus $34.6 \%$; risk ratio $0.77,95 \%$ CI $0.44-1.34$ ) in children or adults, during a follow-up period ranging from 24 to 48 weeks [50-52]. Neither azithromycin nor clarithromycin treatment improved ACQ-7 (mean difference 0.11 points, 95\% CI $-0.34-0.12$ points) or AQLQ (mean difference 0.16 points, $95 \% \mathrm{CI}-0.06-0.37$ points) in adults beyond the MCID.

Relative to placebo, treatment with azithromycin or clarithromycin in adults or children was not associated with changes in post-bronchodilator $\mathrm{FEV}_{1} \%$ pred (mean difference 1.95, 95\% CI -2.42-6.32) or pre-bronchodilator $\mathrm{FEV}_{1} \mathrm{~L}$ (mean difference 0.37, 95\% CI -2.17-2.91) that reached the MCID [50, 53, 54].

The effects of clarithromycin on airway inflammation were inconsistent, with only one of two studies showing significant reductions in airway neutrophilia [55]. Compared with placebo, macrolide therapy in adults was associated with a lower number of lower respiratory tract infections requiring antibiotics $(20.9 \%$ versus $35.6 \%$; risk ratio $0.60,95 \%$ CI $0.45-0.79)$ [50, 51].

The number of study participants with at least one adverse event (67.3\% versus $72.2 \%$; risk ratio $0.93,95 \%$ CI $0.73-1.19)$ and the number of serious adverse events (9.1\% versus $11.4 \%$; risk ratio $0.81,95 \%$ CI $0.52-$ $1.24)$ in adults or children were not different from placebo $[50,51,53,54]$.

\section{Benefits}

Macrolides reduce the number of asthma exacerbations and at least one study suggests that this effect is similar for participants with or without eosinophilia [51]. The effect on asthma control and quality of life does not reach the MCID.

\section{Harms}

Chronic macrolide therapy has been associated with increased incidence of diarrhoea; however, the number of serious adverse events or number of participants with at least one adverse event is not different to placebo. Although macrolides have a potential risk for QT prolongation or hearing loss, the frequency of these events is not reported to be higher than in the placebo arm in patients whom at baseline had no hearing deficits or abnormally prolonged corrected QT [51]. Relative to placebo, the prevalence of nasal and oropharyngeal macrolide-resistant streptococci increased in one study [50], but not in another [51]. Those treated with azithromycin for 48 weeks had reduced airway Haemophilus influenzae load, with no changes to total or pathogenic bacterial loads. Although sputum macrolide resistance genes increased in this group, there was a lower rate of antibiotic use and of adverse events due to clinically diagnosed infections $[51,56]$.

\section{Conclusions and research needs}

Relative to placebo, chronic macrolide therapy reduces the risk of having an asthma exacerbation. However, there is no conclusive evidence that treatment shows any effect in reducing severe exacerbations or hospitalisations. The effects of macrolides on asthma have been limited to participants with uncontrolled or persistently symptomatic disease that may or may not be exacerbation prone; therefore, it is unknown whether this therapy will improve outcomes among those meeting ERS/ATS criteria for severe asthma. The emergence of antimicrobial resistance associated with prolonged antibiotic use such as macrolide therapy is a critical public health issue. Potential benefits in severe asthma need to be carefully considered against this background risk from both the perspective of an individual patient and the wider community.

What others are saying

The 2019 GINA guidelines [20] recommend prescribing add-on low-dose macrolide in patients who do not respond to standard treatment, but classify its use off-label and suggest weighing the benefits against the potential for antibiotic resistance.

In the 2016 BTS/SIGN guidelines [57], the use of macrolide antibiotics in asthma was not recommended; the 2019 BTS/SIGN revised guidelines [39] do not mention the use of chronic macrolide therapy for severe asthma.

The FDA has not approved the use of chronic macrolide therapy for asthma.

ERS/ATS recommendations

We suggest a trial of macrolide treatment to reduce asthma exacerbations in adult asthma subjects on GINA/NAEPP step 5 therapy that remain persistently symptomatic or uncontrolled (conditional recommendation, low quality of evidence) 
We suggest against the use of chronic macrolide treatment in children and adolescents with severe uncontrolled asthma (conditional recommendation, low quality of evidence).

\section{Remarks}

The recommendations are conditional and based on the need to avoid exacerbations and reduce OCS. The benefits and safety of using macrolides for asthma beyond 1 year has not been determined.

\section{Question 6: Should a monoclonal anti-IL-4R $\alpha$ be used in adults and children with severe asthma?} Summary of the evidence

Dupilumab is a fully human monoclonal antibody directed against the $\alpha$ subunit of IL- 4 receptor. It blocks signalling of two key type 2 cytokines: IL-4 and IL-13. We identified three randomised, placebo-controlled trials evaluating dupilumab as add-on therapy in patients with moderate to severe asthma [58-60]. Two randomised controlled trials included adolescent (age 12-17 years) and adult (age $\geqslant 18$ years) participants [59,60], and one trial included only adult participants [58].

In the phase $2 \mathrm{~b}$ dose-ranging clinical trial [58], four dosing regimens of dupilumab were studied: 200 or $300 \mathrm{mg}$ of the drug administered s.c. every 2 or 4 weeks for 24 weeks. 769 adult patients with uncontrolled asthma, despite use of medium- to high-dose ICS and LABA, were randomised 1:1:1:1:1 into four treatment arms or placebo. The primary end-point was change in $\mathrm{FEV}_{1}(\mathrm{~L})$ at 12 weeks in patients with blood eosinophil counts $\geqslant 300 \mu \mathrm{L}^{-1}$. Pre-specified secondary end-points at weeks 12 and 24 included asthma exacerbation rate, time to severe exacerbation, asthma symptom score, asthma quality of life and change in $\mathrm{FEV}_{1}$ (\% pred).

One phase 3 efficacy and safety randomised controlled trial [59] was in adolescents and adults with moderate to severe uncontrolled asthma and it evaluated dupilumab add-on therapy at doses $200 \mathrm{mg}$ (after a loading dose of $400 \mathrm{mg}$ ) or $300 \mathrm{mg}$ (after a loading dose of $600 \mathrm{mg}$ ) every 2 weeks for 52 weeks. A total of 1902 participants were randomised 2:2:1:1 with matched volume placebo. Primary end-points were annualised exacerbation rates (week 52) and absolute change in $\mathrm{FEV}_{1}$ (week 12). Secondary end-points included change in $\mathrm{FEV}_{1} \%$ pred, ACQ and AQLQ, as well as subgroup analysis by blood eosinophil count.

The second phase 3 randomised controlled trial [60] evaluated dupilumab (300 mg every 2 weeks for 24 weeks) in 210 adolescents and adults with severe OCS-dependent asthma. After a steroid dose-optimisation period, patients were randomised 1:1 to receive dupilumab or placebo. OCS dose was adjusted down during weeks 4-20. The primary end-point was percentage reduction in OCS dose required to maintain asthma control. Secondary end-points included proportion of patients with at least $50 \%$ reduction in OCS dose and proportion of patients with reduction in OCS dose to $<5 \mathrm{mg} \cdot \mathrm{day}^{-1}$.

These three trials were pooled for meta-analysis (see evidence profiles in the supplementary material). Effects of dupilumab on exacerbation rate, asthma control, asthma quality of life, lung function and side-effects were assessed for 200 and $300 \mathrm{mg}$ doses at 24 and 52 weeks. Differences in effect size by blood eosinophils were also assessed.

Relative to participants assigned to placebo, those assigned to dupilumab (200 or 300 mg every 2 weeks; 24 and 52 weeks) experienced substantial (46-70.5\%) reductions in annualised rates of asthma exacerbations. Dupilumab therapy resulted in a greater proportion of participants with OCS-dependent severe asthma experiencing $>50 \%$ reduction in OCS dose (relative risk 1.49, 95\% CI 1.22-1.83; absolute risk 26, 95\% CI $12-44$, more achieved $50 \%$ reduction per 100 ), reduction in OCS dose to $<5 \mathrm{mg}^{- \text {day }^{-1}}$ (relative risk 1.92 , 95\% CI 1.46-2.53; absolute risk 344, 95\% CI 172-572, more per 1000) and discontinuation of

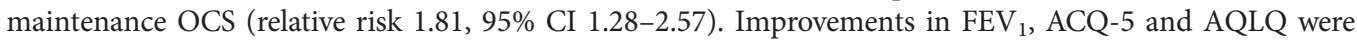
statistically significant, but did not reach the MCID.

The effect size for all above outcomes was larger in patients with blood eosinophil counts $\geqslant 300 \mu \mathrm{L}^{-1}$ when compared with eosinophils $<300 \mu \mathrm{L}^{-1}$ (see evidence profiles in the supplementary material). One study further stratified the study cohort by blood eosinophils $<150,150-300$ and $\geqslant 300 \mu \mathrm{L}^{-1}$ [59]. Rate ratio for annualised severe exacerbation event rate at 52 weeks, pooled for doses 200 and $300 \mathrm{mg}$ every 2 weeks, was 0.33 (95\% CI $0.26-0.42 ; 0.386$ versus 1.158 events.patient ${ }^{-1} \cdot$ year $^{-1}$ ) for the subgroup with blood eosinophils $\geqslant 300 \mu \mathrm{L}^{-1}, \quad 0.60$ (96\% CI $0.43-0.83 ; 0.515$ versus 0.855 events.patient ${ }^{-1}$.year ${ }^{-1}$ ) for the subgroup with blood eosinophils $150-300 \mu \mathrm{L}^{-1}$ and $1.04 \quad(95 \%$ CI $0.76-1.43 ; 0.604$ versus 0.576 events.patient ${ }^{-1}$.year ${ }^{-1}$ ) for the subgroup with blood eosinophils $<150 \mu \mathrm{L}^{-1}$. The same study reported similar results for exacerbations and lung function when stratified by $F_{\mathrm{ENO}} \geqslant 50, \geqslant 25-<50$ and $<25 \mathrm{ppb}$. A post hoc biomarker interaction analysis found the greatest treatment response in patients with $F_{\mathrm{ENO}}$ $\geqslant 25 \mathrm{ppb}$ and blood eosinophils $\geqslant 150 \mu \mathrm{L}^{-1}$. 


\section{Benefits}

Dupilumab, as add-on therapy in patients with asthma that is uncontrolled on medium- to high-dose ICS and LABA, may reduce exacerbations and improve asthma symptoms and lung function. The efficacy is greater in patients with type 2 biomarkers (blood eosinophils $\geqslant 150 \mu \mathrm{L}^{-1}$ or $F_{\mathrm{ENO}} \geqslant 25 \mathrm{ppb}$ ) Dupilumab may reduce OCS dose in patients with severe corticosteroid-dependent asthma.

\section{Harms}

The risk of dupilumab therapy appears to be small, with injection site reaction as the most common treatment-related adverse effect. Frequencies of serious and any side-effects were similar with dupilumab when compared with placebo. However, the mechanisms and potential clinical significance of treatment-related transient blood eosinophilia is not fully understood and needs further elucidation. As dupilumab-mediated eosinophilia has not been associated with adverse events, there are no specific monitoring recommendations.

\section{Conclusions and research needs}

Dupilumab add-on therapy substantially decreases exacerbations in moderate to severe uncontrolled asthma [58-60]. It is effective in reducing OCS dose in patients with severe OCS-dependent asthma. Dupilumab therapy is also associated with improvements in lung function, asthma control and quality of life. More robust improvements were observed in patients with higher eosinophil levels.

Ongoing and future studies should provide additional information on long-term safety and durability of response to dupilumab therapy. More data on efficacy and safety are also needed in children and adolescents. Future studies should also focus on identifying specific disease and population characteristics that can predict response to this therapy.

What others are saying

The 2019 GINA guidelines [20] recommend dupilumab as add-on option for patients with severe eosinophilic or type 2 asthma uncontrolled on high-dose ICS and LABA, or requiring maintenance OCS.

The NICE guidelines [30] do not currently include dupilumab as an add-on therapeutic option for asthma.

\section{ERS/ATS recommendation}

We suggest dupilumab as add-on therapy for adult patients with severe eosinophilic asthma and for those with severe corticosteroid-dependent asthma regardless of eosinophil levels (conditional recommendation).

Remarks

The recommendation places a high value on reducing exacerbations and steroid exposure, and a lower value on cost or burden of the intervention.

The high cost of dupilumab and its impact on cost-effectiveness, equity and feasibility to implementation must be weighed by clinicians in relation to its benefits on asthma outcomes. Due to the limited number of adolescents treated with anti-IL-4/13, the Task Force was unable to provide a recommendation for this age group and no available evidence exists for children $<12$ years of age.

\section{Discussion}

The ERS/ATS Severe Asthma Task Force evaluated six questions that were not addressed in previous guidelines. We conducted a systematic literature search and GRADE analysis to inform recommendations for each specific PICO question regarding the management of severe asthma. The balance of benefits versus burdens, adverse effects and costs, the quality of evidence, and the feasibility and the acceptability were all considered in developing each recommendation (table 2) A conditional recommendation was made for the use of anti-IL-5 and anti-IL-4/13 strategies for severe uncontrolled eosinophilic phenotype. Anti-IL-4/13 is also indicated for systemic corticosteroid-dependent severe asthma subjects regardless of eosinophilic status. Specific eosinophil and $F_{\mathrm{ENO}}$ cut-offs were recommended to identify those with the greatest likelihood of response to anti-IL-5 or anti-IgE therapy. The use of inhaled tiotropium was recommended for adolescents and adults with severe asthma uncontrolled despite GINA step 4-5 or NAEPP step 5 therapies. A trial of chronic macrolide therapy was conditionally suggested to reduce asthma exacerbations in persistently symptomatic or uncontrolled patients on GINA step 5 or NAEPP step 5 therapies. These recommendations should be reconsidered when new evidence becomes available.

It has long been appreciated that the conventional requirements for a good randomised controlled clinical trial do not reflect the reality of patients seen in the clinics [61-63]. Stringent diagnostic requirements are imposed, e.g. adults smokers with asthma are often are excluded to avoid an inadvertent misdiagnosis of 
TABLE 2 European Respiratory Society (ERS)/American Thoracic Society (ATS) Severe Asthma Task Force recommendations for the management of severe asthma

\section{Question Recommendation}

Strength Quality of

evidence

$1 \quad$ We suggest an anti-IL-5 strategy as add-on therapy for adult patients with severe uncontrolled asthma with an eosinophilic phenotype and for those with severe corticosteroid-dependent asthma

2 We suggest that a blood eosinophil cut-point $\geqslant 150 \mu \mathrm{L}^{-1}$ can be used to guide anti-IL-5 initiation in adult patients with severe asthma and a history of prior asthma exacerbations

3

We suggest using a blood eosinophil cut-off $\geqslant 260 \mu \mathrm{L}^{-1}$ to identify adolescents ( $>12$ years) and adults with severe allergic asthma more likely to benefit from anti-lgE treatment

We suggest using a $F_{\text {ENO }}$ cut-off $\geqslant 19.5 \mathrm{ppb}$ to identify adolescents ( $>12$ years) and adults with severe allergic asthma more likely to benefit from anti-lgE treatment

4 For children, adolescents and adults with severe asthma uncontrolled despite GINA step 4-5 or NAEPP step 5 therapies, we recommend the addition of tiotropium

$5 \quad$ We suggest a trial of macrolide treatment to reduce asthma exacerbations in adult asthma subjects on GINA/NAEPP step 5 therapy that remain persistently symptomatic or uncontrolled

We suggest against the use of chronic macrolide treatment in children and adolescents with severe uncontrolled asthma

We suggest dupilumab as add-on therapy for adult patients with severe eosinophilic asthma and for those Conditional with severe corticosteroid-dependent asthma regardless of eosinophil levels

$\begin{array}{cc}\text { Conditional } & \text { Low } \\ \text { Conditional } & \text { Low } \\ \text { Conditional } & \text { Low } \\ \text { Conditional } & \text { Low } \\ \text { Strong } & \text { Moderate } \\ \text { Conditional } & \text { Low } \\ \text { Conditional } & \text { Low } \\ \text { Conditional } & \text { Low }\end{array}$

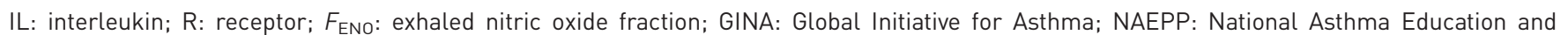
Prevention Program.

COPD. However, this is illogical; nonsmokers also get COPD, and those who smoke and have asthma may be more steroid resistant and thus more, not less likely to profit from biologicals. Frequently there is a requirement for acute bronchodilator reversibility to be demonstrated, even though this is not predictive of a response to treatment and there is no uniform definition.

There could be two reasons for excluding a severe asthmatic patient from a trial of, for example, an anti-type 2 monoclonal [61, 63]. The first entirely logical reason would be the absence of any evidence of type 2 activity; the second, far more dubious, the presence of type 2 activation, but a coexistent disqualification such as smoking or the absence of variable airflow obstruction. The Wessex group recently evaluated 37 randomised controlled trials of type 2 biologicals and found that $<10 \%$ of patients in the Wessex Severe Asthma Cohort would have been enrolled in landmark phase 3 trials of biological therapies, with commonest reasons for exclusion being failure to demonstrate either or both of fixed and variable airflow obstruction [61]. The exclusion rate for patients with eosinophilic asthma was even higher. In the accompanying editorial [64], it was argued that the right approach for future trials of, for example, anti-type 2 strategies would be to include all those with the treatable trait of airway eosinophilia, irrespective of whether there were any other features of asthma present. This is in line with the approach advocated by The Lancet Commission [63] and also the finding of benefit of anti-type 2 strategies in "eosinophilic COPD" $[65,66]$. Fortunately the licensing authorities have taken the approach of focusing on the treatable trait of airway eosinophilia, because otherwise many patients who could benefit would not have access to these medications. It would be important in post-marketing surveillance, which should be mandated for expensive medications, to confirm that features such as smoking and fixed airflow obstruction do not affect response to therapy.

Another important question arising is whether only patients with genuine severe, therapy-resistant asthma should be eligible for biologicals. The initial ERS/ATS Task Force definition, as with so many others, defined severity by the level of prescribed treatment in association with adverse outcomes such as asthma, chronic symptoms and risk [1]. Inherent in the definition is that adherence to medication has been checked and found to be adequate. However, it is increasingly clear that patients prescribed much lower doses of medication are at risk of asthma exacerbations and death. In the UK National Review of Asthma Deaths $[67,68], \sim 60 \%$ of those who died did not meet ERS/ATS criteria for severe asthma. Important factors, as well as the expected positive predictive effect of a previous acute exacerbation, were underuse of ICS, overuse of short-acting $\beta_{2}$-agonists and failure to engage with regular monitoring visits. Severe asthma specialty clinics can help these patients become well controlled by addressing reversible factors such as poor adherence. However, there is a hard core of patients, termed "refractory difficult asthma", who continue with poor adherence and other risk-taking activities despite multiple interventions; in other words, adherence has been optimised as far as possible, but is still inadequate. It has been argued 
elsewhere that such children (or other nonadherent patients) should be offered biologicals if they have the necessary treatable airway trait, to prevent asthma deaths $[69,70]$. The same argument has been advanced in adults. This is not a group that is included in randomised controlled trials, so we cannot make evidence-based recommendations. However, it seems not unreasonable that a persistent treatable trait, whether steroid resistant or uncontrolled because of social factors, should be treated the same way irrespective of cause. However, the condition of giving biologicals to the nonadherent must be that it is directly observed in hospital; such patients cannot be candidates for home therapy.

A future challenge is to ensure that children who might benefit from biologicals actually receive them. There are clear phenotypic differences between paediatric and adult asthma [71], and although atopy is very common in severe paediatric asthma, it is by no means clear that airway eosinophilia is necessarily type 2 driven [72]. Indeed, even in adult asthma, non-type 2 eosinophilic endotypes are being discovered [73]. Also, there is reason to suppose that antieosinophil strategies may be deleterious in children, given the role of the eosinophil in immune homeostasis [74]. There are extensive paediatric data on efficacy and safety of the anti-IgE monoclonal omalizumab [75-77], so there should be no reason not to replicate these studies for other anti-IL-5 strategies, in the absence of a reliable biomarker of efficacy. In summary, it is essential to perform paediatric trials of these new agents that evaluate the impact of these treatments on development and long-term outcomes, and also to pursue research into biomarkers of efficacy [78].

There is another troubling aspect concerning the application of biologicals in children. The conventional sequence of medication testing is in adults first and then, if safety and efficacy is demonstrated, performing studies in children. If there is no efficacy in adults, then the medication is not tested further. An obvious example is the anti-IL-13 monoclonal tralokinumab [79, 80]. At least three randomised controlled studies in adults failed to show significant clinical efficacy [81-83] and there are no plans to do a paediatric trial, on the basis that the data show that the IL-13 pathway is not crucial in airway eosinophilia. It is true that adolescents age $>12$ years are included in these studies, but the actual numbers enrolled are dwarfed by adult participants. Although this seems a logical conclusion in adults, there are no data to confirm or refute this in children; is it conceivable that a potentially valuable paediatric monoclonal has been discarded wrongly? It would be very difficult to prioritise a paediatric tralokinumab trial at present, but it does highlight the need to better understand the similarities and differences between adult and paediatric endotypes.

Although this document has reviewed a large body of high-quality evidence, and highlighted new evidence that OCS dose and asthma exacerbation risk can be substantially reduced, there is much work still to be done. Mepolizumab, benralizumab and reslizumab all target the type 2 pathways, and it is more than likely that further similar compounds will be licensed. The question that arises is how to determine which of an overlapping series of biologicals should be prescribed for the individual patient? Although the majority of studies reviewed here focused on peripheral eosinophils as a marker of type 2 inflammation, other biomarkers such as $F_{\mathrm{ENO}}$ could offer additional information in identifying subendotypes. We speculate that additional type 2 pathway biomarkers will need to be identified in order to do this effectively, and in this regard, the systematic analyses of existing severe asthma cohorts such as SARP (Severe Asthma Research Program) and U-BIOPRED (Unbiased BIOmarkers in PREDiction of respiratory disease outcomes) will be invaluable. Although group data may show one or other is marginally better, it is inconceivable that one will be superior for all individuals. Of course, a series of $n$-of- 1 trials can be carried out, but this is hardly scientific therapeutics. Furthermore, combination of biologicals may prove to be better on the speculation that type 2 inflammation may be most effectively abrogated by blocking all the signature type 2 cytokines, IL-4, IL-5 and IL-13, with dupilumab combined with an anti-IL-5 or anti-IL-5R $\alpha$ strategy. Pragmatic clinical trials may potentially provide answers to these questions for real-life clinical practice [84].

Another future challenge is the role of biologicals in low- and middle-income settings, as the majority of data derive from a developed world setting. There may well be different asthma endotypes across the world and, more importantly, the significance of a raised blood eosinophil count in a region with a high burden of parasitic infections may be different. The World Health Organization defined three groups of severe asthma, of which untreated severe asthma is most relevant to low- and middle-income settings [85]. The first priority must be to ensure that basic asthma medications are uniformly available across the world, which will then enable us to obtain data on the true prevalence of severe, therapy-resistant asthma and refractory difficult asthma in a low- and middle-income setting. The most difficult challenge will be the cost of these medications and making them available to those who would benefit outside a resource-rich area. This challenge is not of course unique to asthma.

Finally, most of the work on the new asthma therapies has been on their role in preventing asthma exacerbations, where they have been very successful. In the future, they may have a role in the aftermath of 
an acute asthma exacerbation. Provided the patient reaches an emergency facility in time, the basic treatment of an asthma exacerbation is straightforward. Much more difficult is to prevent a further exacerbation and it has been highlighted that the period of highest risk is in the month after the signal exacerbation $[67,68]$. Given that outside the pre-school years, asthma exacerbations are caused by respiratory viral infection on the background of uncontrolled type 2-driven airway inflammation, an anti-type 2 strategy as a single injection might well be a promising strategy to reduce relapse, especially as it would not require adherence, and would potentially be efficacious to buy time while other social and environmental factors are addressed. More data are needed before this strategy can be recommended.

In summary, the PICOs studied here have enabled the ERS/ATS Task Force to make recommendations for the treatment of severe asthma, which should lead to modifications of guidelines and improvement in outcomes that are important to patients, namely reduction in OCS dose and exacerbation frequency and improved quality of life. However, we recognise that these recommendations will not be effective across all severe asthma subjects and that more precise phenotype-driven research is needed. We also reiterate that, prior to adopting these novel and in many cases invasive and expensive approaches, every effort should be made to deploy standard medications to maximum benefit. However, for the minority of patients with asthma who, for whatever reason, do not respond to standard therapies and continue to experience frequent exacerbations, we are in an exciting new and evolving world of novel, beneficial approaches.

Acknowledgement: With thanks to the European Lung Foundation severe asthma patient advisory group who provided input on patient views and preferences via teleconferences, through their attendance at the ATS and ERS Congresses, and in writing. They contributed to formulating and prioritising the key questions and outcomes.

This document was endorsed by the ERS Executive Committee on August 26, 2019, and by the ATS on September 14, 2019.

The guidelines published by the European Respiratory Society incorporate data obtained from a comprehensive and systematic literature review of the most recent studies available at the time. Health professionals are encouraged to take the guidelines into account in their clinical practice. However, the recommendations issued by this guideline may not be appropriate for use in all situations. It is the individual responsibility of health professionals to consult other sources of relevant information, to make appropriate and accurate decisions in consideration of each patient's health condition and in consultation with that patient and the patient's caregiver where appropriate and/or necessary, and to verify rules and regulations applicable to drugs and devices at the time of prescription.

Conflict of interest: F. Holguin has nothing to disclose. J.C. Cardet has nothing to disclose. K.F. Chung has received honoraria for participating in advisory board meetings of GlaxoSmithKline, AstraZeneca, Novartis, Merck, Boehringer Ingelheim, 4D Pharma, Pieris and Teva regarding treatments for asthma, cough and chronic obstructive pulmonary disease and has also been remunerated for speaking engagements. S. Diver has nothing to disclose. D.S. Ferreira reports fellowship MTF 2015-02 from the European Respiratory Society, during the conduct of the study. A. Fitzpatrick has nothing to disclose. M. Gaga reports grants and personal fees from AstraZeneca and Chiesi, grants from Novartis, Menarini and Elpen, personal fees from MSD and BMS, outside the submitted work. L. Kellermeyer has nothing to disclose. S. Khurana reports grants from GlaxoSmithKline and Sanofi, outside the submitted work. S. Knight has nothing to disclose. V.M. McDonald reports grants from GlaxoSmithKline, grants and personal fees for lectures from AstraZeneca, personal fees for educational steering committee work from Menarini, outside the submitted work. R.L. Morgan has nothing to disclose. V.E. Ortega has nothing to disclose. D. Rigau acts as a European Respiratory Society methodologist. P. Subbarao has nothing to disclose. T. Tonia acts as a European Respiratory Society methodologist. I.M. Adcock has nothing to disclose. E.R. Bleecker undertaken clinical trials through his employer, Wake Forest School of Medicine and University of Arizona, for AstraZeneca, MedImmune, Boehringer Ingelheim, Genentech, Johnson and Johnson (Janssen), Novartis, Regeneron and Sanofi Genzyme, and has also served as a paid consultant for AstraZeneca, MedImmune, Boehringer Ingelheim, GlaxoSmithKline, Novartis, Regeneron and Sanofi Genzyme, outside the submitted work. C. Brightling reports grants and personal fees from GlaxoSmithKline, Novartis, Genentech/Roche, Chiesi, 4D Pharma, Glenmark, Boehringer Ingelheim, Mologic, Gossamer and AstraZeneca/MedImmune, personal fees from Sanofi/Regeneron and Teva, outside the submitted work. L-P. Boulet reports research grants for participation in multicentre studies from AstraZeneca, Boston Scientific, GlaxoSmithKline, Hoffman La Roche, Novartis, Ono Pharma, Sanofi and Takeda; support for research projects submitted by the investigator from AstraZeneca, Boehringer Ingelheim, GlaxoSmithKline, Merck and Takeda; consulting and advisory board work for AstraZeneca, GlaxoSmithKline, Novartis and Methapharm; nonprofit grants for production of educational materials from AstraZeneca, Boehringer Ingelheim, GlaxoSmithKline, Merck Frosst and Novartis; conference fees from AstraZeneca, GlaxoSmithKline, Merck and Novartis; support for participation in conferences and meetings from Novartis and Takeda. M. Cabana reports personal fees for consultancy from Genentech and Novartis, outside the submitted work. M. Castro receives university grant funding from NIH, American Lung Association and PCORI; receives pharmaceutical grant funding from AstraZeneca, Boehringer Ingelheim, Chiesi, GlaxoSmithKline, Novartis and Sanofi-Aventis; is a consultant for Aviragen, Boston Scientific, Genentech, Nuvaira, Neutronic, Therabron, Theravance, Vectura, 4D Pharma, VIDA, Mallinckrodt, Teva and Sanofi-Aventis; is a speaker for AstraZeneca, Boehringer Ingelheim, Boston Scientific, Genentech, Regeneron, Sanofi and Teva; and receives royalties from Elsevier. P. Chanez has undertaken consultancy services for Boehringer Ingelheim, GlaxoSmithKline, AstraZeneca, Novartis, Teva, Chiesi, Sanofi and SNCF, served on advisory boards for Boehringer Ingelheim, GlaxoSmithKline, AstraZeneca, Novartis, Teva, Chiesi and Sanofi, received lecture fees from GlaxoSmithKline, AstraZeneca, Novartis, Teva, Chiesi, Boston Scientific and ALK, and received industry-sponsored grants from AstraZeneca, ALK and Novartis. A. Custovic reports personal fees for consultancy from Novartis, Regeneron/Sanofi, Boehringer Ingelheim and Philips, personal fees for lectures from Thermo Fisher Scientific and Novartis, outside the submitted work. R. Djukanovic reports receiving fees for lectures at symposia organised by 
Novartis, AstraZeneca and Teva, consultation for Teva and Novartis as member of advisory boards, and participation in a scientific discussion about asthma organised by GlaxoSmithKline; and is a co-founder of and current consultant for, and has shares in, Synairgen, a University of Southampton spin-out company. U. Frey reports personal fees for meeting attendance from GlaxoSmithKline Scientific Advisory Board, outside the submitted work. B. Frankemölle has nothing to disclose. P. Gibson reports grants and personal fees for educational activities from GlaxoSmithKline, grants and personal fees for lectures and educational activities from AstraZeneca, personal fees for educational steering committee work from Novartis and Sanofi, outside the submitted work. D. Hamerlijnck is independent European Federation of Allergy and Airways (EFA) patient advisor to Novartis on patient involvement; patient co-chair of the ERS Severe Heterogeneous Asthma Registry, Patient-centred CRC; member of the scientific advisory board of the Veelbelovende Zorg for ZIN and ZonMw; member of the scientific advisory board of the BENEFIT (ZonMw-KBC Dutch Belgian collaboration); member of the IMI PARADIGM patient advisory group for EPF; member of the scientific advisory board for ERA-NET NEURON Biomarkers; patient reviewer for the Dutch Patient Federation and several ZonMw scientific committees. N. Jarjour reports the following, outside the submitted work: a grant from National Institutes of Health; personal fees for consultation from AstraZeneca and Boehringer Ingelheim. S. Konno reports grants from AstraZeneca, Kyorin Pharmaceutical, Japan Allergy Foundation, Novartis and Ministry of Education, Culture, Sports, Science and Technology of Japan, during the conduct of the study. H. Shen has nothing to disclose. C. Vitari has nothing to disclose. A. Bush has nothing to disclose.

Support statement: This work was supported by the European Respiratory Society and the American Thoracic Society. Funding information for this article has been deposited with the Crossref Funder Registry.

\section{References}

1 Chung KF, Wenzel SE, Brozek JL, et al. International ERS/ATS guidelines on definition, evaluation and treatment of severe asthma. Eur Respir J 2014; 43: 343-373.

2 Guyatt GH, Oxman AD, Vist GE, et al. GRADE: an emerging consensus on rating quality of evidence and strength of recommendations. BMJ 2008; 336: 924-926.

3 Wedzicha J, Miravitlles M, Hurst JR, et al. Management of COPD exacerbations: a European Respiratory Society/ American Thoracic Society guideline. Eur Respir J 2017; 49: 1600791.

4 Wedzicha JA, Calverley PMA, Albert RK, et al. Prevention of COPD exacerbations: a European Respiratory Society/American Thoracic Society guideline. Eur Respir J 2017; 50: 1602265.

5 Santanello NC, Zhang J, Seidenberg B, et al. What are minimal important changes for asthma measures in a clinical trial? Eur Respir J 1999; 14: 23-27.

6 Juniper EF, Svensson K, Mork AC, et al. Measurement properties and interpretation of three shortened versions of the asthma control questionnaire. Respir Med 2005; 99: 553-558.

7 Juniper EF, Guyatt GH, Willan A, et al. Determining a minimal important change in a disease-specific quality of life questionnaire. J Clin Epidemiol 1994; 47: 81-87.

8 Bel EH, Wenzel SE, Thompson PJ, et al. Oral glucocorticoid-sparing effect of mepolizumab in eosinophilic asthma. N Engl J Med 2014; 371: 1189-1197.

9 Ortega HG, Liu MC, Pavord ID, et al. Mepolizumab treatment in patients with severe eosinophilic asthma. $N$ Engl J Med 2014; 371: 1198-1207.

10 Chupp GL, Bradford ES, Albers FC, et al. Efficacy of mepolizumab add-on therapy on health-related quality of life and markers of asthma control in severe eosinophilic asthma (MUSCA): a randomised, double-blind, placebo-controlled, parallel-group, multicentre, phase 3b trial. Lancet Respir Med 2017; 5: 390-400.

11 Bjermer L, Lemiere C, Maspero J, et al. Reslizumab for inadequately controlled asthma with elevated blood eosinophil levels: a randomized phase 3 study. Chest 2016; 150: 789-798.

12 Castro M, Mathur S, Hargreave F, et al. Reslizumab for poorly controlled, eosinophilic asthma: a randomized, placebo-controlled study. Am J Respir Crit Care Med 2011; 184: 1125-1132.

13 Castro M, Zangrilli J, Wechsler ME, et al. Reslizumab for inadequately controlled asthma with elevated blood eosinophil counts: results from two multicentre, parallel, double-blind, randomised, placebo-controlled, phase 3 trials. Lancet Respir Med 2015; 3: 355-366.

14 Corren J, Weinstein S, Janka L, et al. Phase 3 study of reslizumab in patients with poorly controlled asthma: effects across a broad range of eosinophil counts. Chest 2016; 150: 799-810.

15 Bleecker ER, FitzGerald JM, Chanez P, et al. Efficacy and safety of benralizumab for patients with severe asthma uncontrolled with high-dosage inhaled corticosteroids and long-acting $\beta_{2}$-agonists (SIROCCO): a randomised, multicentre, placebo-controlled phase 3 trial. Lancet 2016; 388: 2115-2127.

16 Castro M, Wenzel SE, Bleecker ER, et al. Benralizumab, an anti-interleukin 5 receptor $\alpha$ monoclonal antibody, versus placebo for uncontrolled eosinophilic asthma: a phase $2 \mathrm{~b}$ randomised dose-ranging study. Lancet Respir Med 2014; 2: 879-890.

17 FitzGerald JM, Bleecker ER, Nair P, et al. Benralizumab, an anti-interleukin-5 receptor $\alpha$ monoclonal antibody, as add-on treatment for patients with severe, uncontrolled, eosinophilic asthma (CALIMA): a randomised, double-blind, placebo-controlled phase 3 trial. Lancet 2016; 388: 2128-2141.

18 Nair P, Wenzel S, Rabe KF, et al. Oral glucocorticoid-sparing effect of benralizumab in severe asthma. $N$ Engl J Med 2017; 376: 2448-2458.

19 Park HS, Kim MK, Imai N, et al. A phase 2a study of benralizumab for patients with eosinophilic asthma in South Korea and Japan. Int Arch Allergy Immunol 2016; 169: 135-145.

20 Global Initiative for Asthma. Global Strategy for Asthma Management and Prevention. 2019. http://ginasthma.org/ 2019-gina-report-global-strategy-for-asthma-management-and-prevention Date last accessed: October 28, 2019.

21 National Institute for Health and Care Excellence. Mepolizumab for Treating Severe Refractory Eosinophilic Asthma. TA431. 2017. www.nice.org.uk/guidance/ta431 Date last accessed: October 1, 2019.

22 National Institute for Health and Care Excellence. Reslizumab for Treating Severe Eosinophilic Asthma. TA479. 2017. www.nice.org.uk/guidance/ta479 Date last accessed: October 1, 2019.

23 National Institute for Health and Care Excellence. Benralizumab for Treating Severe Eosinophilic Asthma. TA565. 2019. www.nice.org.uk/guidance/ta565 Date last accessed: October 1, 2019. 
24 Institute for Clinical and Economic Review. Biologic Therapies for Treatment of Asthma Associated with Type 2 Inflammation: Effectiveness, Value, and Value-Based Price Benchmarks. 2018. https://icer-review.org/wp-content/ uploads/2018/04/ICER_Asthma_Draft_Report_092418v1.pdf Date last accessed: October 1, 2019.

25 Nair P, Pizzichini MM, Kjarsgaard M, et al. Mepolizumab for prednisone-dependent asthma with sputum eosinophilia. N Engl J Med 2009; 360: 985-993.

26 Ortega HG, Yancey SW, Mayer B, et al. Severe eosinophilic asthma treated with mepolizumab stratified by baseline eosinophil thresholds: a secondary analysis of the DREAM and MENSA studies. Lancet Respir Med 2016; 4: 549-556.

27 Mukherjee M, Aleman Paramo F, Kjarsgaard M, et al. Weight-adjusted intravenous reslizumab in severe asthma with inadequate response to fixed-dose subcutaneous mepolizumab. Am J Respir Crit Care Med 2018; 197: 38-46.

28 Khatri S, Moore W, Gibson PG, et al. Assessment of the long-term safety of mepolizumab and durability of clinical response in patients with severe eosinophilic asthma. J Allergy Clin Immunol 2019; 143: 1742-1751.

29 Busse WW, Bleecker ER, FitzGerald JM, et al. Long-term safety and efficacy of benralizumab in patients with severe, uncontrolled asthma: 1-year results from the BORA phase 3 extension trial. Lancet Respir Med 2019; 7: 46-59.

30 National Institute for Health and Care Excellence. Asthma: Diagnosis, Monitoring and Chronic Asthma Management. 2017. www.nice.org.uk/guidance/ng80 Date last accessed: October 28, 2019.

31 Ledford D, Busse W, Trzaskoma B, et al. A randomized multicenter study evaluating Xolair persistence of response after long-term therapy. J Allergy Clin Immunol 2017; 140: 162-169.

32 Hanania NA, Wenzel S, Rosen K, et al. Exploring the effects of omalizumab in allergic asthma: an analysis of biomarkers in the EXTRA study. Am J Respir Crit Care Med 2013; 187: 804-811.

33 Busse W, Spector S, Rosen K, et al. High eosinophil count: a potential biomarker for assessing successful omalizumab treatment effects. J Allergy Clin Immunol 2013; 132: 485-486.

34 Izuhara K, Ohta S, Ono J. Using periostin as a biomarker in the treatment of asthma. Allergy Asthma Immunol Res 2016; 8: 491-498.

35 Casale TB, Chipps BE, Rosen $\mathrm{K}$, et al. Response to omalizumab using patient enrichment criteria from trials of novel biologics in asthma. Allergy 2018; 73: 490-497.

36 Busse W, Corren J, Lanier BQ, et al. Omalizumab, anti-IgE recombinant humanized monoclonal antibody, for the treatment of severe allergic asthma. J Allergy Clin Immunol 2001; 108: 184-190.

37 Soler M, Matz J, Townley R, et al. The anti-IgE antibody omalizumab reduces exacerbations and steroid requirement in allergic asthmatics. Eur Respir J 2001; 18: 254-261.

38 Humbert M, Taille C, Mala L, et al. Omalizumab effectiveness in patients with severe allergic asthma according to blood eosinophil count: the STELLAIR study. Eur Respir J 2018; 51: 1702523.

39 British Thoracic Society, Scottish Intercollegiate Guidelines Network. British Guideline on the Management of Asthma. SIGN 158. 2019. www.sign.ac.uk/sign-158-british-guideline-on-the-management-of-asthma.html Date last accessed: October 28, 2019.

40 Kerstjens HA, Disse B, Schroder-Babo W, et al. Tiotropium improves lung function in patients with severe uncontrolled asthma: a randomized controlled trial. J Allergy Clin Immunol 2011; 128: 308-314.

41 Kerstjens HA, Engel M, Dahl R, et al. Tiotropium in asthma poorly controlled with standard combination therapy. N Engl J Med 2012; 367: 1198-1207.

42 Hamelmann E, Bernstein JA, Vandewalker M, et al. A randomised controlled trial of tiotropium in adolescents with severe symptomatic asthma. Eur Respir J 2017; 49: 1601100

43 Kerwin E, Wachtel A, Sher L, et al. Efficacy, safety, and dose response of glycopyrronium administered by metered dose inhaler using co-suspension delivery technology in subjects with intermittent or mild-to-moderate persistent asthma: a randomized controlled trial. Respir Med 2018; 139: 39-47.

44 Lee LA, Briggs A, Edwards LD, et al. A randomized, three-period crossover study of umeclidinium as monotherapy in adult patients with asthma. Respir Med 2015; 109: 63-73.

45 Lee LA, Yang S, Kerwin E, et al. The effect of fluticasone furoate/umeclidinium in adult patients with asthma: a randomized, dose-ranging study. Respir Med 2015; 109: 54-62.

46 Peters SP, Bleecker ER, Kunselman SJ, et al. Predictors of response to tiotropium versus salmeterol in asthmatic adults. J Allergy Clin Immunol 2013; 132: 1068-1074.

47 Kerstjens HA, Moroni-Zentgraf P, Tashkin DP, et al. Tiotropium improves lung function, exacerbation rate, and asthma control, independent of baseline characteristics including age, degree of airway obstruction, and allergic status. Respir Med 2016; 117: 198-206.

48 Szefler SJ, Murphy K, Harper T 3rd, et al. A phase III randomized controlled trial of tiotropium add-on therapy in children with severe symptomatic asthma. J Allergy Clin Immunol 2017; 140: 1277-1287.

49 National Asthma Education and Prevention Program. Guidelines for the Diagnosis and Management of Asthma. EPR-3. 2007. www.nhlbi.nih.gov/health-topics/guidelines-for-diagnosis-management-of-asthma Date last accessed: October 28, 2019.

50 Brusselle GG, Vanderstichele C, Jordens P, et al. Azithromycin for prevention of exacerbations in severe asthma (AZISAST): a multicentre randomised double-blind placebo-controlled trial. Thorax 2013; 68: 322-329.

51 Gibson PG, Yang IA, Upham JW, et al. Effect of azithromycin on asthma exacerbations and quality of life in adults with persistent uncontrolled asthma (AMAZES): a randomised, double-blind, placebo-controlled trial. Lancet 2017; 390: 659-668.

52 Strunk RC, Bacharier LB, Phillips BR, et al. Azithromycin or montelukast as inhaled corticosteroid-sparing agents in moderate-to-severe childhood asthma study. J Allergy Clin Immunol 2008; 122: 1138-1144.

53 Hahn DL, Grasmick M, Hetzel S, et al. Azithromycin for bronchial asthma in adults: an effectiveness trial. J Am Board Fam Med 2012; 25: 442-459.

54 Sutherland ER, King TS, Icitovic N, et al. A trial of clarithromycin for the treatment of suboptimally controlled asthma. J Allergy Clin Immunol 2010; 126: 747-753.

55 Simpson JL, Powell H, Boyle MJ, et al. Clarithromycin targets neutrophilic airway inflammation in refractory asthma. Am J Respir Crit Care Med 2008; 177: 148-155.

56 Taylor SL, Leong LEX, Mobegi FM, et al. Long-term azithromycin reduces Haemophilus influenzae and increases antibiotic resistance in severe asthma. Am J Respir Crit Care Med 2019; 200: 309-317. 
British Thoracic Society, Scottish Intercollegiate Guidelines Network. 2016. British Guideline on the Management of Asthma. SIGN 153. www.brit-thoracic.org.uk/quality-improvement/guidelines/asthma Date last accessed: October 28, 2019.

58 Wenzel S, Castro M, Corren J, et al. Dupilumab efficacy and safety in adults with uncontrolled persistent asthma despite use of medium-to-high-dose inhaled corticosteroids plus a long-acting $\beta_{2}$ agonist: a randomised double-blind placebo-controlled pivotal phase $2 \mathrm{~b}$ dose-ranging trial. Lancet 2016; 388: 31-44.

59 Castro M, Corren J, Pavord ID, et al. Dupilumab efficacy and safety in moderate-to-severe uncontrolled asthma. $N$ Engl J Med 2018; 378: 2486-2496.

60 Rabe KF, Nair P, Brusselle G, et al. Efficacy and safety of dupilumab in glucocorticoid-dependent severe asthma. $N$ Engl J Med 2018; 378: 2475-2485.

61 Brown T, Jones T, Gove K, et al. Randomised controlled trials in severe asthma: selection by phenotype or stereotype. Eur Respir J 2018; 52: 1801444.

62 Agusti A, Bel E, Thomas M, et al. Treatable traits: toward precision medicine of chronic airway diseases. Eur Respir J 2016; 47: 410-419.

63 Pavord ID, Beasley R, Agusti A, et al. After asthma: redefining airways diseases. Lancet 2018; 391: 350-400.

64 Shrimanker R, Beasley R, Kearns C. Letting the right one in: evaluating the generalisability of clinical trials. Eur Respir J 2018; 52: 1802218.

65 Pavord ID, Chanez P, Criner GJ, et al. Mepolizumab for eosinophilic chronic obstructive pulmonary disease. $N$ Engl J Med 2017; 377: 1613-1629.

66 Kerkhof M, Sonnappa S, Postma DS, et al. Blood eosinophil count and exacerbation risk in patients with COPD. Eur Respir J 2017; 50: 1700761.

67 Levy ML, Winter R. Asthma deaths: what now? Thorax 2015; 70: 209-210.

68 Royal College of Physicians. National Review of Asthma Deaths 2012-13. 2015. www.rcplondon.ac.uk/projects/ national-review-asthma-deaths Date last accessed: October 1, 2019.

69 Bush A, Saglani S, Fleming L. Severe asthma: looking beyond the amount of medication. Lancet Respir Med 2017; 5: 844-846.

70 Green RH, Shaw D. Strict adherence rules to obtain monoclonal therapy might cost lives. Lancet Respir Med 2017; 5: 678-679.

71 Andersson CK, Adams A, Nagakumar P, et al. Intraepithelial neutrophils in pediatric severe asthma are associated with better lung function. J Allergy Clin Immunol 2017; 139: 1819-1829.

72 Bossley CJ, Fleming L, Gupta A, et al. Pediatric severe asthma is characterized by eosinophilia and remodeling without $\mathrm{T}_{\mathrm{H}} 2$ cytokines. J Allergy Clin Immunol 2012; 129: 974-982.

73 Kuo CS, Pavlidis S, Loza M, et al. T-helper cell type 2 (Th2) and non-Th2 molecular phenotypes of asthma using sputum transcriptomics in U-BIOPRED. Eur Respir J 2017; 49: 1602135.

74 Travers J, Rothenberg ME. Eosinophils in mucosal immune responses. Mucosal Immunol 2015; 8: 464-475.

75 Normansell R, Walker S, Milan SJ, et al. Omalizumab for asthma in adults and children. Cochrane Database Syst Rev 2014; 1: CD003559.

76 Corren J, Kavati A, Ortiz B, et al. Efficacy and safety of omalizumab in children and adolescents with moderate-to-severe asthma: a systematic literature review. Allergy Asthma Proc 2017; 38: 250-263.

77 Odajima H, Ebisawa M, Nagakura T, et al. Long-term safety, efficacy, pharmacokinetics and pharmacodynamics of omalizumab in children with severe uncontrolled asthma. Allergol Int 2017; 66: 106-115.

78 Saglani S, Bush A, Carroll W, et al. Biologics for paediatric severe asthma: trick or TREAT? Lancet Respir Med 2019; 7: 294-296.

79 Nair P, O'Byrne PM. The interleukin-13 paradox in asthma: effective biology, ineffective biologicals. Eur Respir J 2019; 53: 1802250

80 Chung KF. Tralokinumab unsuccessful for management of severe, uncontrolled asthma. Lancet Respir Med 2018; 6: 480-481.

81 Busse WW, Brusselle GG, Korn S, et al. Tralokinumab did not demonstrate oral corticosteroid-sparing effects in severe asthma. Eur Respir J 2019; 53: 1800948.

82 Russell RJ, Chachi L, FitzGerald JM, et al. Effect of tralokinumab, an interleukin-13 neutralising monoclonal antibody, on eosinophilic airway inflammation in uncontrolled moderate-to-severe asthma (MESOS): a multicentre, double-blind, randomised, placebo-controlled phase 2 trial. Lancet Respir Med 2018; 6: 499-510.

83 Panettieri RA Jr, Sjobring U, Peterffy A, et al. Tralokinumab for severe, uncontrolled asthma (STRATOS 1 and STRATOS 2): two randomised, double-blind, placebo-controlled, phase 3 clinical trials. Lancet Respir Med 2018; 6: 511-525.

84 Pilette C, Brightling C, Lacombe D, et al. Urgent need for pragmatic trial platforms in severe asthma. Lancet Respir Med 2018; 6: 581-583.

85 Bousquet J, Mantzouranis E, Cruz AA, et al. Uniform definition of asthma severity, control, and exacerbations: document presented for the World Health Organization Consultation on Severe Asthma. J Allergy Clin Immunol 2010; 126: 926-938. 